\title{
The Science Case for LIGO-India
}

\author{
M. Saleem ${ }^{1,7}$, Javed Rana ${ }^{9}$, V. Gayathri ${ }^{5,6}$, Aditya Vijaykumar ${ }^{2}$, \\ Srashti Goyal ${ }^{2}$, Surabhi Sachdev ${ }^{9}$, Jishnu Suresh ${ }^{11}$, S. \\ Sudhagar ${ }^{4}$, Arunava Mukherjee ${ }^{8}$, Gurudatt Gaur ${ }^{12}$, Bangalore \\ Sathyaprakash $^{9}$, Archana Pai ${ }^{5}$, Rana X Adhikari ${ }^{2,3}$, P. Ajith ${ }^{2}$, \\ Sukanta Bose B $^{40}$
}

${ }^{1}$ Chennai Mathematical Institute, Siruseri 603103, Tamilnadu, India

${ }^{2}$ International Centre for Theoretical Sciences, Tata Institute of Fundamental

Research, Bangalore 560089, India

${ }^{3}$ LIGO Laboratory, California Institute of Technology, USA

${ }^{4}$ Inter-University Centre for Astronomy and Astrophysics (IUCAA), Post Bag 4, Ganeshkhind, Pune 411 007, India

${ }^{5}$ Department of Physics, Indian Institute of Technology Bombay, Powai, Mumbai 400 076, India

${ }^{6}$ Department of Physics, University of Florida, PO Box 118440, Gainesville, FL 32611-8440, USA

${ }^{7}$ School of Physics and Astronomy, University of Minnesota, Minneapolis, MN 55455, USA

${ }^{8}$ Saha Institute of Nuclear Physics, HBNI, 1/AF Bidhannagar, Kolkata-700064, India

${ }^{9}$ Institute for Gravitation and the Cosmos, The Pennsylvania State University, University Park, PA 16802, USA

${ }^{10}$ Department of Physics and Astronomy, Washington State University, 1245 Webster, Pullman, WA 99164-2814, USA

${ }^{11}$ Institute for Cosmic Ray Research (ICRR), The University of Tokyo, Kashiwa City, Chiba 277-8582, Japan

${ }^{12}$ Institute of Advanced Research, Gandhinagar 382 426, Gujarat, India

E-mail: sukanta@iucaa.in

4 January 2022

\begin{abstract}
The global network of gravitational-wave detectors has completed three observing runs with $\sim 50$ detections of merging compact binaries. A third LIGO detector, with comparable astrophysical reach, is to be built in India (LIGO-Aundha) and expected to be operational during the latter part of this decade. Such additions to the network increase the number of baselines and the network SNR of GW events. These enhancements help improve the sky-localization of those events. Multiple detectors simultaneously in operation will also increase the baseline duty factor, thereby, leading to an improvement in the detection rates and, hence, the completeness of surveys. In this paper, we quantify the improvements due to the expansion of the LIGO Global Network (LGN) in the precision with which source properties will be measured. We also present examples of how this expansion will give a boost to tests of fundamental physics.
\end{abstract}




\section{Contents}

1 Introduction $\quad 3$

1.1 Detectors . . . . . . . . . . . . . . . . . . . . 3

1.2 Simulations ........................... 4

2 CBC Detection rates $\quad 5$



2.2 Improvement in the effective duty-factor of a network . . . . . . . . 6

2.3 Detection rates . . . . . . . . . . . . . . . . . . . 7

3 Parameter estimation $\quad 8$

3.1 Improvement in errors for binary black hole events . . . . . . . . . . . . . 9 9

3.1.1 Sky-localization: . . . . . . . . . . . . . . 10

3.1.2 Luminosity distance and inclination angle: . . . . . . . . . . . 10

3.1 .3 Source masses: . . . . . . . . . . . . . . . . 11

3.2 Improved measurements of matter effects: source classification and BNS

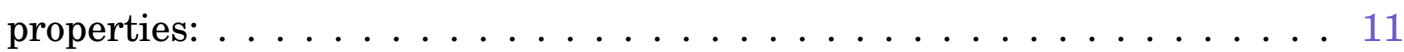

4 Sky localization and early warning $\quad 14$

4.1 Early warning of binary neutron star mergers . . . . . . . . . . 17

5 Tests of GR $\quad 20$

5.1 Improved constraints on deviations from GR . . . . . . . . . . . . . 20

5.2 Constraints on the nature of $\mathrm{GW}$ polarisations . . . . . . . . . . . . . 21

6 Conclusions and summary 22 


\section{Introduction}

The global network of gravitational-wave $(\mathrm{GW})$ detectors (comprising the two LIGO interferometers [1] and the Virgo interferometer [2]) has completed three observing runs with 50 detections of merging compact binaries [3]. A fourth detector in Japan [4] is now being commissioned and is expected to join the global network in 2022. A third LIGO detector with comparable astrophysical reach is being built in India [5] and is expected to be operational during the latter part of this decade. Several detectors operating in different parts of the globe provide multiple long baselines and an increased network SNR. These characteristics help improve the sky-localization of GW events, among other things [6]. Multiple detectors operating simultaneously will also improve the duty factor of the network leading to improvements in the detection rates.

In this paper we quantify the improvements arising due to the addition of a LIGO detector in India to the LIGO Global Network (LGN). The global GW detector network will include, additionally, Virgo and KAGRA, further enhancing the improvements described herein. In this work, we choose to focus on the LGN to understand the improvement in the network during times when Virgo and KAGRA are not taking data. We quantitatively describe how this leads to better astrophysical insights about the source properties and how that improves our ability to probe fundamental physics and cosmological models. We find that the addition of a new detector in India brings substantial benefits to the scientific capabilities of the LGN.

\subsection{Detectors}

The LGN will consist of 3 interferometers in the upgraded configuration of Advanced LIGO (so-called A+) [7], with the third detector in Aundha, in the Hingoli district, in the eastern part of the state of Maharashtra, India. It is expected that the two LIGO detectors in the U.S. will be upgraded into this configuration in $~ 2026$ and that the detector in Aundha will come online soon after. Following the existing naming convention $\neq$ the detector in India will be referred to as LIGO-Aundha (A). The LIGO Global Network with and without LIGO-Aundha will be denoted as AHL and HL, respectively. Our studies below compare their performances, mainly related to the compact binary coalescence searches. Networks involving additional detectors will likely see further improvement in performances than what is found here. Moreover, the involvement of other detectors may reduce the impact of the improvements that the addition of LIGO-Aundha alone would bring. The broader study is, however, beyond the scope of this work.

$\ddagger$ where the detectors are named after the nearby town; LIGO-Hanford $(\mathrm{H})$ and LIGO-Livingston (L) 


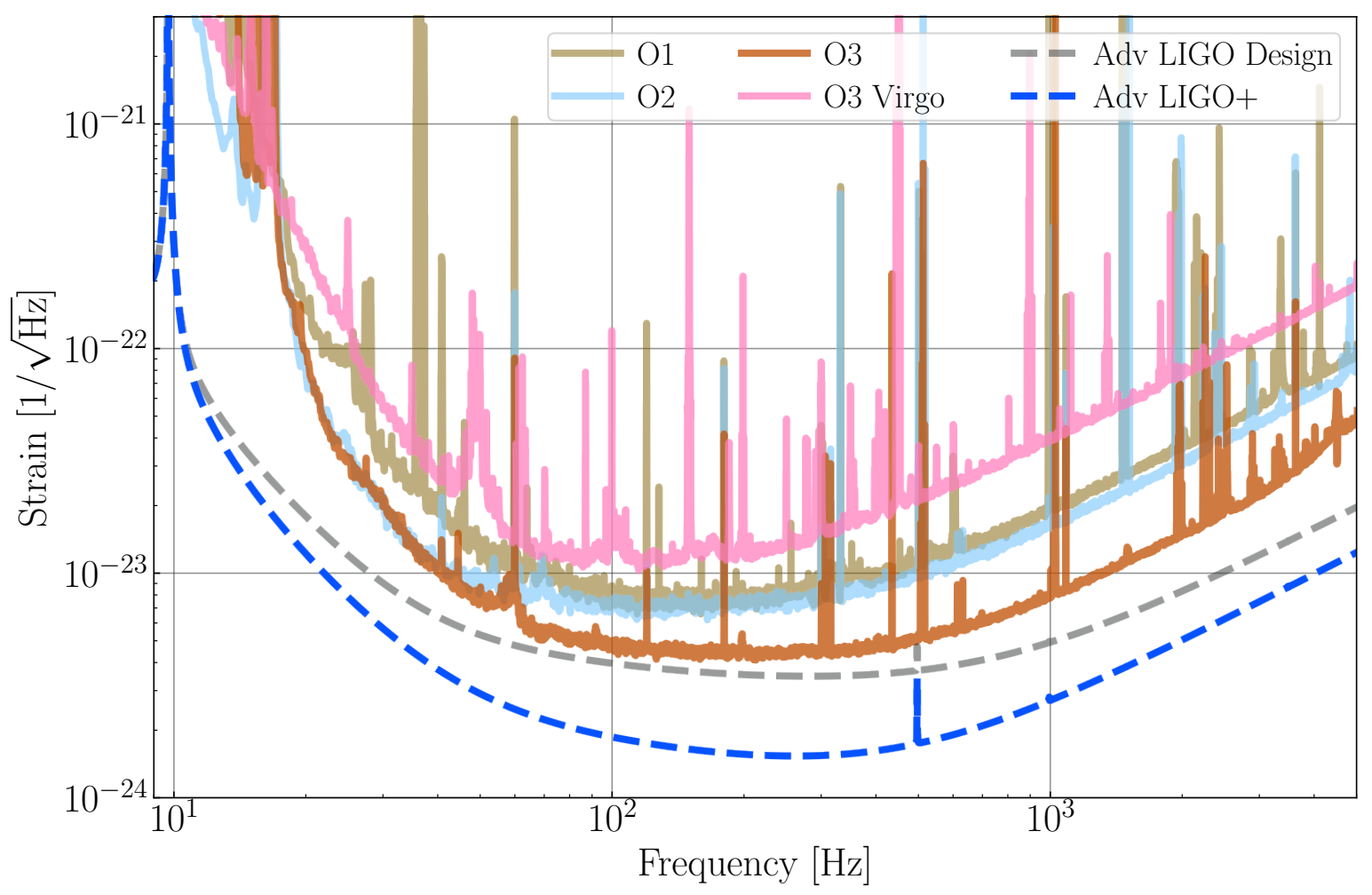

Figure 1: Strain noise spectral density of the LIGO Interferometers during the observing runs O1-O3. Also shown are the Virgo O3 noise, the Advanced LIGO design sensitivity, and the A+ sensitivity for the LIGO detectors Aundha, Hanford, and Livingston (labeled as "Adv LIGO+").

\subsection{Simulations}

The compact binary coalescences (CBCs) observed in the $\mathrm{GW}$ window range in total mass from $3-150 M_{\odot}$. While most of the binary systems harbor primary objects with masses $<45 M_{\odot}$, a few systems have the primary heavier than $45 M_{\odot}$. The recently released gravitational-wave transient catalog (GWTC-2) considers several population mass distribution models to obtain the merger rates [3, 8]. For the simulations in this study, we use one of those mass models with the model parameters taken from the observed binary black-hole mergers [8]. In this model, the primary mass follows a power-law distribution with some spectral index up to a certain maximum mass and a uniform Gaussian component with a finite width to account for high masses, together with a smoothing function at low masses to avoid a hard cut-off. The mass ratio follows a smoothed power-law distribution. We choose the median values of the hyper-parameters of these distributions inferred in [8] for simulations.

Astrophysical models suggest that binary black holes with isotropically distributed component spins can form in dense environments, such as globular clusters and galactic centers. At the same time, we expect black-hole spins to get aligned with the orbital angular momentum in isolated binaries [9, 10]. The black hole spin distribution uses a 
model that is a mixture of both these possibilities [8]. We use this model for drawing the spins of both the compact objects in a binary for our simulations. Besides, the binary sources are oriented uniformly and distributed uniformly over the sky and placed uniformly in co-moving volume up to a red-shift of 1.5 using the Planck 2015 cosmology [11].

The binary black hole $(\mathrm{BBH})$ simulations described above are used in various studies below on quantifying the improvement in the performance of the network arising from its expansion to include LIGO-Aundha. These include a discussion of $\mathrm{BBH}$ detection rates in Section 2 and quantifying the improvement in the estimation of binary parameters in Section 3. In Section 4, we discuss the possibility of sending early warning alerts to electromagnetic and particle observatories before the epoch of binary coalescence. We make projections in Section 5 on how a detected $\mathrm{BBH}$ population can be used to place observational bounds on deviations from General Relativity (GR).

\section{CBC Detection rates}

Coalescing compact binaries involving neutron stars and black holes are, so far, the only GW sources detected in past GW observing runs [12, 3]. The inclusion of LIGO-Aundha in the LGN will boost the rate at which we detect such binaries. This enhancement will arise owing to improved sky-coverage, distance reach, and baseline duty factor, which is the effective observation period of a detector network. In this section, we quantitatively assess the improvement in the $\mathrm{CBC}$ detection rate $\left(R_{\mathrm{det}}\right)$ for $\mathrm{AHL}$ vis à vis the HL network.

We focus here on the stellar-mass $\mathrm{BBHs}$, which are the main contributor to the menagerie of signals observed by LIGO-Virgo so far. Our analysis can be straightforwardly extended to classes of CBC sources that involve neutron stars.

For an astrophysical population of $\mathrm{BBHs}$ with a comoving constant merger-rate density $r_{\text {merg }}$ in units of $\mathrm{Gpc}^{-3} \mathrm{yr}^{-1}$, the detection rate (per year) is given by $R_{\text {det }}=$ $r_{\text {merg }} \times\langle V T\rangle$, where $\langle V T\rangle$ is the population-marginalized detection volume averaged over the period of observation for any given detector network (for more details, see $[3,13]$ and the references therein). The assumption that $r_{\text {merg }}$ is non-evolving w.r.t. redshift is a simplified assumption and hence could affect the rates we reported in this paper, however it has negligible impact on the rates comparison between two networks which is the goal of this study. The factor $\langle V T\rangle$ crucially depends on the number of detectors, their sensitivity as well as the search methodologies and their ability to treat the non-Gaussian noisy transients in the multi-detector data. 


\subsection{Detection criteria}

We simulate the noise in any detector as Gaussian, with a vanishing mean, and uncorrelated with the noise in any other detector. $\S$ Then the network coherent SNR-squared is the sum of the SNR-squared of signals in the individual detectors $[18,19]$. Below we discuss two alternative criteria for assessing whether a signal can be considered as detected by a network (similar considerations are made in [20]):

(i) Coherent network SNR criterion: For an $N$-detector network, this criterion is $\sqrt{\sum_{k=1}^{N} \rho_{k}^{2}} \geq \rho_{\text {thresh }}^{\text {net }}$, where $\rho_{k}$ is the SNR at the $k^{t h}$ detector. Here we set the threshold of $\rho_{\text {thresh }}^{\text {net }}$ to a value that keeps the false-alarm probability associated with it low enough to make a confident detection case.

(ii) Multi-detector coincidence criterion: $\sqrt{\sum_{k=1}^{N} \rho_{k}^{2}} \geq \rho_{\text {thresh }}^{\text {net }}$ and $\rho_{k}>4$ for at least two of the $N$ detectors.

For the LGN studied here we set $\rho_{\text {thresh }}^{\text {net }}=12$, which is conservative in the sense that there have been detections with two or three detectors with network SNR below 12. We present search performance metrics for both criteria below.

Arguably, the simplest way to identify interesting detection candidates is to apply the first criterion. Its biggest advantage is that it allows for picking up sources that are loud enough in one detector but weak in the others, e.g., if located in their blind-spots. This can happen since no two detectors have the same orientation. Nevertheless, this criterion has a few limitations: For instance, a loud noise-transient in a single detector (e.g, non-Gaussian glitches) can give rise to a trigger that satisfies this network criterion and, therefore, gets misclassified as a detection candidate. On the other hand, if one requires that at least two detectors record a high enough SNR, such as what the second criterion above employs, then the false-alarm rate reduces significantly (such as by mitigating the effects of non-Gaussian glitches), albeit by sacrificing some degree of sky coverage (figure 1 ).

\subsection{Improvement in the effective duty-factor of a network}

Duty factor of a detector (network) is defined as the fraction of clock time for which the detector (network) acquires science quality data. Assuming that each detector in the network has a duty factor of $d_{f}$, one can analytically compute the effective duty factor $\mathrm{d}_{f \text { eff }}$ for each multi-detector network. For the multi-detector coincidence criterion, $\mathrm{d}_{f \text { eff }}$ is the fraction of the observation period during which at least two of the detectors

$\S$ This is a simplification since real detector noise contains non-Gaussian transients, which contribute to the background rate. Still modeling detector noise as Gaussian is useful. As has been demonstrated in multiple LIGO-Virgo CBC and detector characterization papers [14, 15, 16, 17], glitch classification and mitigation techniques have achieved some degree of success in cleaning the background to make it largely Gaussian-like. Simulation studies, like ours, are not the first ones, and are useful also for providing targets and benchmarks for those data quality/cleaning efforts. It is for these reasons Gaussian studies remain relevant. 

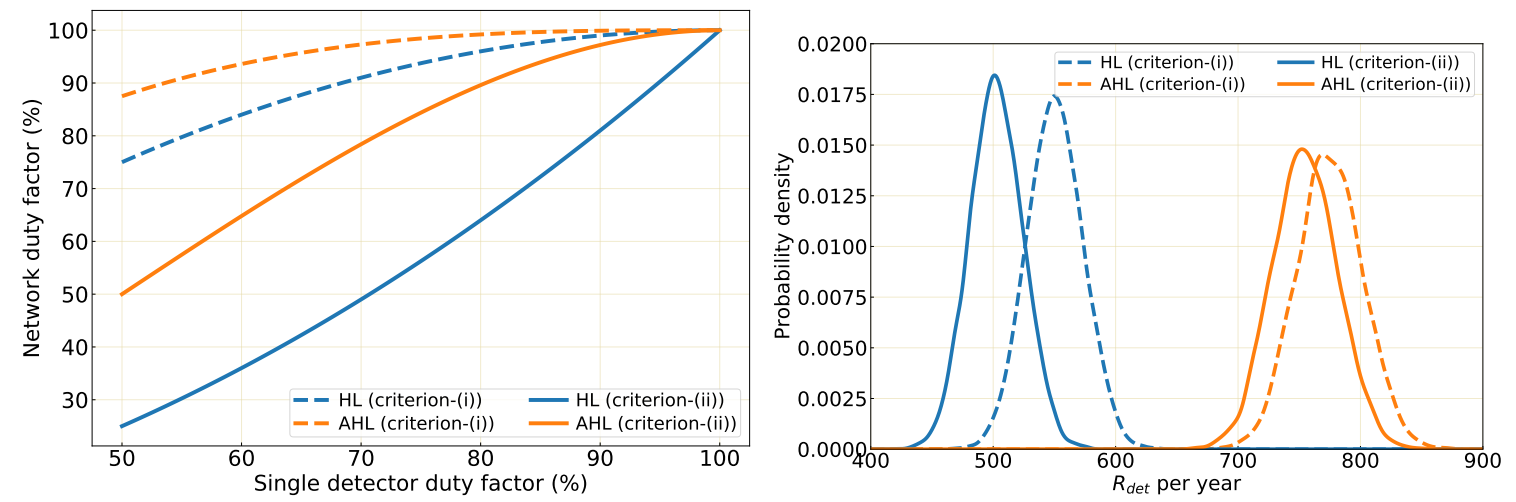

Figure 2: [Left] Network duty factors of the HL and AHL networks as functions of the single-detector duty factor. [Right] The distribution of the detection rates of stellarmass binary black-hole detection rates for the same two networks using the GWTC-2 population models.

are simultaneously collecting science-quality data while for the network SNR criterion, it is the fraction of observing period when at least one of the detectors is observing in science mode. For an $N$-detector network, the effective duty factor is

$$
\mathrm{d}_{f \text { eff }}=\sum_{k=N_{\min }}^{N}{ }^{N} C_{k} d_{f}^{k}\left(1-d_{f}\right)^{N-k},
$$

where the summation runs from $k=N_{\min }$ to $k=N$ with $N_{\text {min }}$ being the minimum number of detectors required by the coincidence criterion. Specifically, we have $N_{\min }=$ 1 for criterion-(i) and $N_{\text {min }}=2$ for criterion-(ii). The combinatorics symbol ${ }^{N} C_{k}$ denotes the number of possible unique $k$-detector combinations one can form in a network of $N$ detectors. Figure 2 shows how the effective duty factor improves with the addition of LIGO-Aundha. Assuming 90\% single-detector duty-factor, the AHL duty-factor gets boosted by a factor of 1.2 compared to the HL network if one follows criterion-(ii), while the improvement is only one per-cent under criterion-(i); see, e.g., Figure 2. ॥

\subsection{Detection rates}

We perform extensive simulations to estimate the detection rates of merging compact binaries for the HL and AHL configurations. The published detections of binary black hole mergers provide an up to date median BBH merger rate density of $r_{\text {merg }}=23 \mathrm{Gpc}^{-3}$ $\mathrm{yr}^{-1}$ [8]. With this rate density and a uniform source distribution in the comoving volume, we populate $\sim 8684$ sources up to a redshift of 1.5 . II We perform 8000

\| Here it is assumed that the unlocked time-stretches are randomly and uniformly distributed over the full observation period, which excludes any stretches of time scheduled for concurrent downtime for all detectors.

II It is a somewhat arbitrary choice that we truncate the population at a maximum redshift of 1.5. However, this is motivated by the fact that at higher redshifts, the actual comoving rate density could significantly be different from the merger rate density at $z=0$ (the one we assumed in this study) 


\begin{tabular}{|l|c|c|}
\hline Network & Criterion (i) & Criterion (ii) \\
\hline HL & $550.0_{-29.0}^{+30.0}$ & $502.0_{-27.0}^{+29.0}$ \\
AHL & $775.0_{-35.0}^{+35.0}$ & $754.0_{-33.0}^{+35.0}$ \\
\hline
\end{tabular}

Table 1: Detection rates (in $\mathrm{yr}^{-1}$ ) of stellar-mass binary black holes in HL and AHL networks, assuming A+ sensitivity and a duty factor of $90 \%$ for every detector.

batches of simulations, with each batch containing 8684 sources with the mass and spin distributions following the ones detailed in Section 1.2. We further distribute the sources uniformly over the sky with the binary orientation distributed uniformly. For all the sources, we apply the detection criteria (i) and (ii) and obtain the detection rates $\left(R_{\text {det }}\right)$. The right panel of Figure 2 provides the distribution of the estimated $R_{\text {det }}$.

Table 1 provides the detection rate estimates of $\mathrm{BBH}$ for HL and AHL network configurations assuming 90\% single-detector duty factor. Compared to HL, the detection rate in AHL increases by $41 \%$ and $50 \%$ for criteria (i) and (ii), respectively. Besides the duty factor, the sky coverage of the two networks determines their detection rates. Since the coincidence criterion-(ii) exhibits a preference for shortlisting highly significant events, one can expect that with LIGO-Aundha one will see a perceptible increase in such events under that criterion.

\section{Parameter estimation}

With the expansion of the LIGO global network and the consequent enhancement in the signal-to-noise ratios of the CBC detections and mitigation of parameter degeneracies, one would anticipate improvements in the astrophysical parameter estimation. In this section, we employ CBC signal simulations to obtain quantitative support for this expectation.

For a $\mathrm{BBH}$ system in a circular orbit, the gravitational-wave signal is characterized by component masses $\left(m_{1}, m_{2}\right)$, component spins $\left(\vec{S}_{1}, \vec{S}_{2}\right)$, the luminosity distance $\left(D_{L}\right)$, orbital inclination angle $(\iota)$, polarisation angle $(\psi)$, sky-position angles $(\alpha, \delta)$ and the coalescence time and phase $\left(t_{c}, \phi_{c}\right)$. For a binary neutron star (BNS) system, we require at least two additional parameters in the form of component tidal deformability parameters $\left(\Lambda_{1}, \Lambda_{2}\right)$.

The CBC signal's multi-dimensional parameter space harbors correlations and degeneracies among different parameter pairs, contributing to the uncertainties in the measurements of the individual parameters. For several of these parameters, the errorbar scales inversely with the signal-to-noise ratio (for loud signals) [21]. While this holds particularly well for the intrinsic binary parameters, such as component masses and spins, the aforementioned degeneracies among some pairs, e.g., (i) the sky-location angles $\alpha$ and $\delta$ and (ii) $d_{L}$ and $\iota$, can not often be removed despite high SNR. The due to the star formation rate as well as the distribution of delay time between the formation and the coalescence of the binary. 
expansion of LGN with LIGO-Aundha, in addition to increasing the SNR, will enhance parameter estimation accuracy by providing an independent observation of the source that can significantly reduce the degeneracies among some of the parameters.

In Sec. 3.1 we focus on general parameter estimation for select BBH events, and in Sec. 3.2 we present the primary results of masses and tidal effects in BNS systems.

\subsection{Improvement in errors for binary black hole events}

For this study, we simulated binary black hole signals modeled after two of the observed binary black holes, namely, (i) the loudest BBH, GW150914 [22] and (ii) the most massive BBH, GW190521 [23]. In fact, GW150914 is the first binary black hole merger observed by two LIGO detectors and the loudest event so far, with a coherent SNR of 24. The observed component masses were $36 M_{\odot}$ and $29 M_{\odot}$ with a remnant $\mathrm{BH}$ of $62 M_{\odot}$, and the event was located at a luminosity distance of $450 \mathrm{Mpc}$. It was localized in a huge sky-patch, spanning 590 sq. degs.

GW190521 is the most massive and among the farthest $(5 \mathrm{Gpc})$ binary black hole mergers observed so far. Its component masses are $85 M_{\odot}$ and $66 M_{\odot}$. The remnant was estimated to have a mass of $142 M_{\odot}$. This is the first intermediate-mass BH candidate observed in the gravitational-wave window.

For our two simulations, the injected values of the key parameters are listed in Table 2 where we choose the masses and spins to be identical to those inferred for GW150914 and GW190521. From our 8000 batches of BBH simulations described in Sec. 2, it was found that the population-averaged ratio of SNR at AHL to the SNR at HL lies in the range of $1.3-1.4$. We choose the injected sky positions in such a way that the SNR at AHL is 1.4 times the SNR at the HL so that it resembles the average behaviour of SNR improvement.

The run-of-the-mill Bayesian parameter estimation approach assumes stationary Gaussian detector noise and a reliable, faithful Einstein's GR signal model for the GW signal from the compact binary merger. An up to date suite of models for complete CBC waveforms constructed by combining various approaches include phenomenological models, such as IMRPhenom models [24], the effective one-body EOBNR waveforms that use inputs from numerical relativity [25, 26, 27], and the NRSurrogates waveforms derived from numerical relativity simulations [28, 29, 30]. In our analysis, we use the IMRPhenomPv2 [31, 32, 33, 34, 35] waveform model for both injections as well as recovery. We use the Bilby [36] software package, with its in-built sampler dynesty, to perform the parameter estimation. We perform this analysis with zero-noise signal injections ${ }^{+}$and the likelihood computed using the A+ PSD.

We tabulate the results in terms of improvement in the $90 \%$ credible intervals on various astrophysical parameters in Table 2 and present pictorially in Fig. 3 the posterior probability contours (at $90 \%$, and $68 \%$ credible levels). In that figure, the left and right panels depict the results for the GW150914- and GW190521-like injections,

+ A zero-noise signal injection refers to data that has only a simulated GW signal and no added noise. 
respectively.

\begin{tabular}{|c|c|c|c|c|}
\hline \multirow{2}{*}{ Parameter } & \multicolumn{2}{|c|}{ GW150914-like } & \multicolumn{2}{c|}{ GW190521-like } \\
\cline { 2 - 5 } & Injected & Improvement & Injected & Improvement \\
\hline \hline Chirpmass $\left(M_{\odot}\right)$ & 28.1 & $33 \%$ & 64.6 & $39 \%$ \\
Total mass $\left(M_{\odot}\right)$ & 65.0 & $33 \%$ & 149.6 & $40 \%$ \\
$D_{L}$ in Gpc & 2.5 & $35 \%$ & 5.3 & $36 \%$ \\
$\iota$ in deg. & 45 & $27 \%$ & 45 & $70 \%$ \\
Sky localization in deg $^{2}$. & & $92 \%$ & & $96 \%$ \\
\hline
\end{tabular}

Table 2: Parameter estimation improvement in $90 \%$ credible intervals in expanding the network from HL to AHL: We use BBH signals modelled after GW150914 and GW190521 and estimate the improvement in sky-localization, luminosity distance, binary inclination, masses and spins. The imporvemnt for a parameter $X$ is defined as $\left(\left(\Delta X_{A H L}-\Delta X_{H L}\right) / \Delta X_{H L}\right) \times 100$ where $\Delta X$ is the $90 \%$ credible error bar.

3.1.1. Sky-localization: The detector pair comprising LIGO-Aundha and LIGOLivingston provides the longest baseline amongst all pairs of existing / in-construction detectors. This improves the precision with which sources can be localized in the sky. For the GW150914-like injection, the 90\% credible 2-D localization area is $\sim 114$ $\operatorname{deg}^{2}$ which improves to $\sim 9 \mathrm{deg}^{2}$ with AHL. This amounts to $92 \%$ reduction in the localization uncertainty. For GW190521-like injection, we find a $\sim 96 \%$ reduction, with the respective localization area for HL and AHL configurations being $\sim 971 \mathrm{deg}^{2}$ and $\sim 35 \mathrm{deg}^{2}$. More discussion on the sky-localization can be found in Sec. 4 and the reader may also refer to earlier studies on localization, e.g., Refs. [37, 38] and the references therein.

3.1.2. Luminosity distance and inclination angle: The three-detector configuration plays a crucial role in breaking the degeneracy between the luminosity distance $D_{L}$ and the inclination angle $\iota$. For the GW150914-like system, the errors in $D_{L}$ and the inclination shrink by $35 \%$ and $27 \%$, respectively, for AHL relative to HL. Similarly, for the GW190521-like injection the error reduction in the same parameters is $36 \%$ and $70 \%$, respectively. The improved distance estimates also benefit from the reduced $2 \mathrm{D}$ sky-localization of the source by the AHL network since, aided by an improved network SNR, it helps break the degeneracy between distance and sky position. This will have direct implications in the measurements of cosmological parameters [39, 40, 41, 42, 43, 44]. We also find significant improvement in the inclination angle measurement of the binary $(\iota)$ which is partly due to the resolution of the distance-inclination degeneracy. Though this analysis has been performed on binary black hole mergers, similar improvements are expected in the inclination angles of binary neutron stars and neutron star-black hole mergers as well [45] which are 
favourite candidates to have associated EM counterparts. Accurate knowledge of the inclination angle is key in doing multimessenger astronomy, for making predictions on the possible EM counterparts and in understanding the physical process that drives the EM counterparts [46, 47, 48]. Further, improved precision in the binary inclination helps to probe the gravitational-wave polarisation of the signal. This improvement directly impacts probing alternative theories of gravity with gravitational wave signals. In Sec. 5.2 we discuss how polarisation measurements benefit from the expansion of LGN.

3.1.3. Source masses: Source-frame masses are defined as the detector-frame masses divided by a factor $(1+z)$, where $z$ is the source redshift, which in turn can reveal the source luminosity distance given a cosmological model. Therefore, the measurement of source-frame masses benefits from both the improved SNR and the improved luminosity distance measurement. For the GW150914-like injection, the errors in both the source-frame chirpmass and total mass improve by $\sim 33 \%$. Similarly, for the GW190521-like injection, these improvements are 39\% and 40\%, respectively. See Fig. 3 for the $m_{1}-m_{2}$ contour plots for both the events. Accurate knowledge of the intrinsic source parameters helps in the population synthesis studies of compact binary mergers and obtain constraints on the merger rate density [8].

\subsection{Improved measurements of matter effects: source classification and BNS properties:}

Binary neutron stars are characterized by the masses $\left(m_{1}, m_{2}\right)$ and the tidal deformability parameters $\left(\Lambda_{1}, \Lambda_{2}\right)$ [49] of their components. The presence of matter is predominantly captured by the effective tidal deformability parameter $(\widetilde{\Lambda})$ which is defined by a suitable combination of $m_{1}, m_{2}, \Lambda_{1}$ and $\Lambda_{2}$ [50]. Black holes in general relativity are predicted to have zero tidal deformability, i.e., $\Lambda_{1}=\Lambda_{2}=0$. For a $\mathrm{BBH}$ system, this leads to $\widetilde{\Lambda}=0$ irrespective of their component masses and spins. Moreover, precise estimation of the tidal deformability parameters can constrain the theoretically proposed equations of state of neutron stars and, thus, shed light on the nature of their internal composition [51, 52, 53].

Here we illustrate how the addition of the LIGO-Aundha detector can potentially impact our ability to constrain the effective tidal deformability parameter $(\widetilde{\Lambda})$ as well as discriminate it from the $\widetilde{\Lambda}=0$ case corresponding to BBHs. We do so by employing a fully Bayesian statistical framework [54].

We analyze a set of simulated BNS events with source properties consistent with the first BNS event, GW170817 [51, 54]. Although the chirp-mass $\left(M_{c}\right)$ was very well determined to be $1.188 M_{\odot}$, the component masses have broader uncertainties due to the less precisely measured mass-ratio parameter (e.g., the symmetric mass-ratio, $\eta$ ). Moreover, although GW170817 could successfully rule out the stiffest equations of state, it still has sufficiently broader uncertainty in estimated tidal deformability 

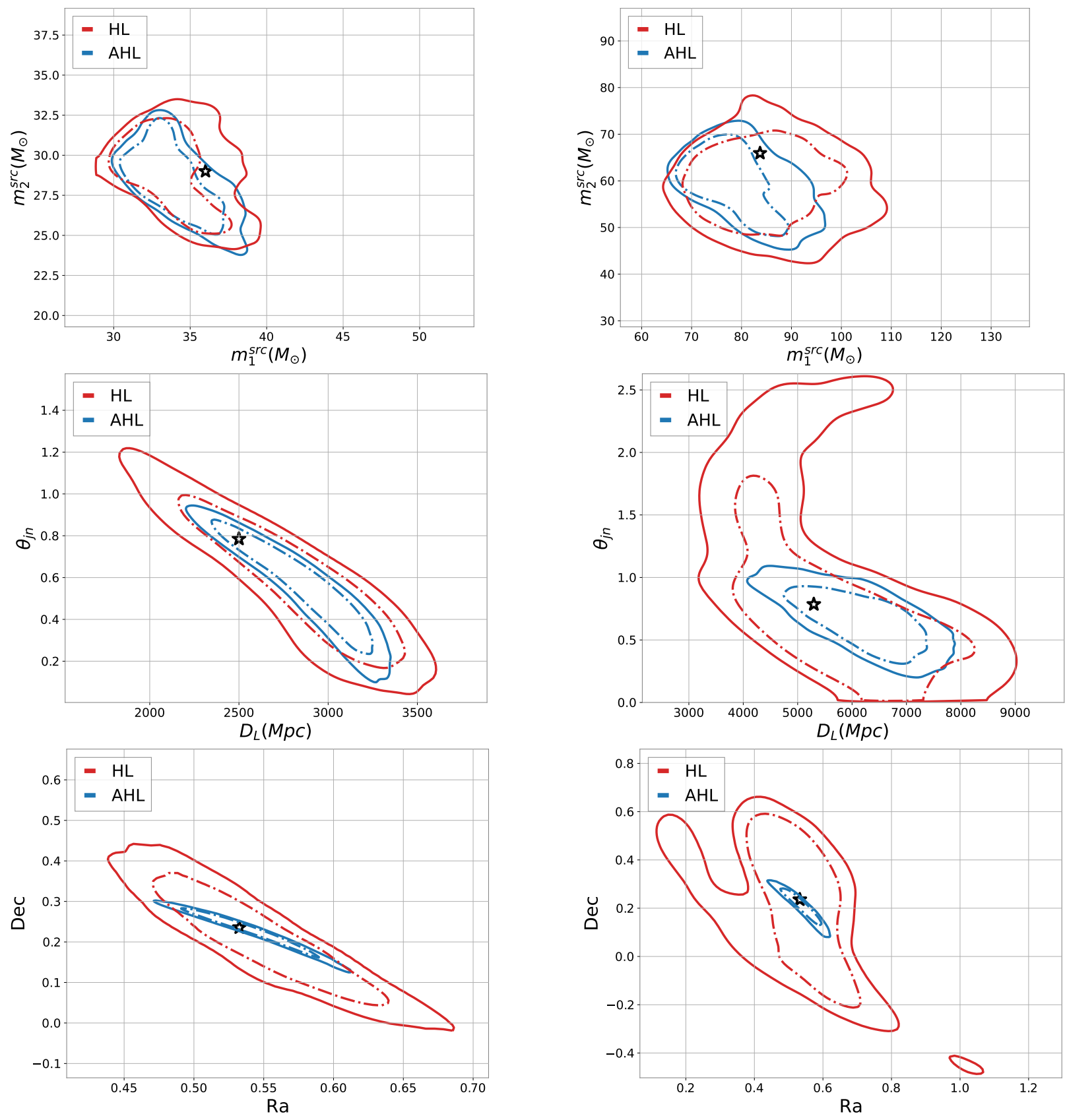

Figure 3: Posterior distributions of certain parameters for GW150914-like (left) and GW190521-like (right) simulated signals in the HL and AHL networks: Top, middle and bottom panels correspond to the parameters $m_{1}^{s r c}-m_{2}^{s r c}, D_{L}-\iota$ and $R A-D e c$, respectively. The true values are shown by a black star. The $95 \%$ and $65 \%$ confidence intervals are shown by solid and dash-dotted lines, respectively.

parameters, thereby, leaving a wide variety of neutron star EOSs viable.

We perform a systematic injection study of Bayesian parameter estimation for a set of simulated signals from BNS events covering the extreme corners of the parameter space, comprising component masses and tidal deformability parameters that are 


\begin{tabular}{|c|c|c|c|c|c|c|c|c|c|}
\hline \multicolumn{3}{|c|}{ Source parameters } & \multirow[b]{2}{*}{ Network } & \multicolumn{3}{|c|}{ Measurement accuracies } & \multicolumn{3}{|c|}{ Improvements in \% } \\
\hline $\begin{array}{c}m_{1}, m_{2} \\
\left(M_{\odot}\right)\end{array}$ & $\Lambda_{1}, \Lambda_{2}[\widetilde{\Lambda}]$ & $\begin{array}{c}D_{L} \\
(\mathrm{Mpc})\end{array}$ & & $\begin{array}{l}\Delta M_{c} \\
\left(M_{\odot}\right)\end{array}$ & $\Delta \eta$ & $\Delta \widetilde{\Lambda}$ & $\begin{array}{c}\Delta M_{c} \\
\text { (in \%) }\end{array}$ & $\begin{array}{c}\Delta \eta \\
\text { (in } \% \text { ) }\end{array}$ & $\begin{array}{c}\Delta \widetilde{\Lambda} \\
\text { (in \%) }\end{array}$ \\
\hline $1.35,1.35$ & $400,400[400]$ & 40 & $\begin{array}{c}\mathrm{LH} \\
\mathrm{AHL}\end{array}$ & $\begin{array}{l}9.7 e-5 \\
7.4 e-5\end{array}$ & $\begin{array}{l}6.8 \mathrm{e}-3 \\
5.8 \mathrm{e}-3\end{array}$ & $\begin{array}{c}131.9 \\
99.6\end{array}$ & 23.7 & 14.7 & 24.5 \\
\hline $1.35,1.35$ & $857,857[857]$ & 40 & $\begin{array}{c}\mathrm{LH} \\
\mathrm{AHL}\end{array}$ & $\begin{array}{l}9.8 \mathrm{e}-5 \\
8.3 \mathrm{e}-5\end{array}$ & $\begin{array}{l}6.8 \mathrm{e}-3 \\
5.8 \mathrm{e}-3\end{array}$ & $\begin{array}{l}156.9 \\
115.2\end{array}$ & 15.3 & 14.7 & 26.6 \\
\hline $1.60,1.17$ & $120,980[551.5]$ & 40 & $\begin{array}{c}\mathrm{LH} \\
\mathrm{AHL}\end{array}$ & $\begin{array}{l}1.3 \mathrm{e}-4 \\
1.1 \mathrm{e}-4\end{array}$ & $\begin{array}{l}9.6 \mathrm{e}-3 \\
8.3 \mathrm{e}-3\end{array}$ & $\begin{array}{l}147.9 \\
110.9\end{array}$ & 15.4 & 13.5 & 25.5 \\
\hline $1.35,1.35$ & $400,400[400]$ & 100 & $\begin{array}{c}\mathrm{LH} \\
\mathrm{AHL}\end{array}$ & $\begin{array}{l}1.6 \mathrm{e}-4 \\
1.3 \mathrm{e}-4\end{array}$ & $\begin{array}{l}7.6 \mathrm{e}-3 \\
5.6 \mathrm{e}-3\end{array}$ & $\begin{array}{l}269.0 \\
217.7\end{array}$ & 18.8 & 16.3 & 19.1 \\
\hline $1.35,1.35$ & $857,857[857]$ & 100 & $\begin{array}{c}\mathrm{LH} \\
\mathrm{AHL}\end{array}$ & $\begin{array}{l}1.7 \mathrm{e}-4 \\
1.4 \mathrm{e}-4\end{array}$ & $\begin{array}{l}7.5 \mathrm{e}-3 \\
6.7 \mathrm{e}-3\end{array}$ & $\begin{array}{l}418.7 \\
218.6\end{array}$ & 17.6 & 10.6 & 47.8 \\
\hline $1.60,1.17$ & 120,980 [551.5] & 100 & $\begin{array}{c}\text { LH } \\
\text { AHL }\end{array}$ & $\begin{array}{l}1.9 \mathrm{e}-4 \\
1.6 \mathrm{e}-4\end{array}$ & $\begin{array}{l}1.0 \mathrm{e}-2 \\
9.0 \mathrm{e}-3\end{array}$ & $\begin{array}{l}384.2 \\
215.0\end{array}$ & 15.8 & 10.0 & 44.1 \\
\hline $1.35,1.35$ & $400,400[400]$ & 250 & $\begin{array}{c}\mathrm{LH} \\
\mathrm{AHL}\end{array}$ & $\begin{array}{l}4.7 \mathrm{e}-4 \\
3.5 \mathrm{e}-4\end{array}$ & $\begin{array}{l}8.5 \mathrm{e}-3 \\
7.6 \mathrm{e}-3\end{array}$ & $\begin{array}{c}1141.5 \\
675.4\end{array}$ & 25.5 & 10.6 & 40.8 \\
\hline $1.35,1.35$ & 857,857 [857] & 250 & $\begin{array}{c}\mathrm{LH} \\
\mathrm{AHL}\end{array}$ & $\begin{array}{l}3.5 \mathrm{e}-4 \\
2.8 \mathrm{e}-4\end{array}$ & $\begin{array}{l}6.9 \mathrm{e}-3 \\
5.7 \mathrm{e}-3\end{array}$ & $\begin{array}{c}1298.6 \\
588.5\end{array}$ & 20.0 & 17.4 & 54.7 \\
\hline $1.60,1.17$ & 120,980 [551.5] & 250 & $\begin{array}{c}\mathrm{LH} \\
\mathrm{AHL}\end{array}$ & $\begin{array}{l}3.2 \mathrm{e}-4 \\
2.9 \mathrm{e}-4\end{array}$ & $\begin{array}{l}1.0 \mathrm{e}-2 \\
9.0 \mathrm{e}-3\end{array}$ & $\begin{array}{l}2264.7 \\
638.51\end{array}$ & 9.4 & 10.0 & 69.9 \\
\hline
\end{tabular}

Table 3: This table summarizes comparisons of key properties of binary neutron star mergers events with high and relatively low-SNR events. Each of the BNS events is simulated with two neutron star EOSs, one softer (SLy4) and one stiffer $(\mathrm{BHB} \Lambda \phi)$. The uncertainties in mass parameters, namely in chirp-mass $\left(\Delta M_{c}\right)$ and symmetric mass-ratio $(\Delta \eta)$ as well as uncertainties in the effective tidal deformability parameter $(\Delta \widetilde{\Lambda})$ of the BNS systems are quoted with $90 \%$ credible level (see subsection 3.2 ). The percentage improvements are quntified following the expresion in Table 2 caption.

consistent with GW170817. Initially, we consider all the sources to be located at a luminosity distance $\left(D_{L}\right)$ of $40 \mathrm{Mpc}$ (similar to the GW170817 event). We consider one equal-mass BNS $\left(m_{1}=m_{2}=1.35 M_{\odot}\right)$ and one unequal-mass $\operatorname{BNS}\left(m_{1}=1.60 M_{\odot}, m_{2}=\right.$ $1.17 M_{\odot}$ ), with soft EOS, namely SLy4 [55], consistent with the GW170817 observation. For the equal-mass case, we also consider the possibility that the neutron stars have a stiff EOS, namely, BHB $\Lambda \phi[56]$.

Given the current estimation of BNS merger event rates (see Sec. 2), it is improbable that such an event will be observed at a distance $D_{L} \lesssim 40 \mathrm{Mpc}$ in the near future. We, therefore, perform additional simulations with the entire set of events (a) at $D_{L}=100 \mathrm{Mpc}$ as well as (b) at $D_{L}=250 \mathrm{Mpc}$. In our simulations, we use IMRPHENOMDNRTIDAL waveform model [57] for the coalescing BNS systems with slow $\left(\left|s_{1}\right|,\left|s_{2}\right| \leq 0.05\right)$, in-plane component spinning configurations for simplicity since astronomical distributions demonstrate that more rapidly spinning BNS systems 
are rare as well as this configuration captures the key aspects reasonably well. We summarize the measurements of mass and tidal deformability parameters with $90 \%$ Bayesian uncertainty intervals in table 3.2.

This study demonstrates that for the very high SNR events (with comparable single detector SNRs 110 - 130 in each of the H, L, and A detectors) the improvement of precision in $M_{c}$ is in the range of $15-25 \%$ and in $\eta$ is of about $10-17 \%$ for the AHL-network of detectors as compared to the HL-network. The improvement in $\widetilde{\Lambda}$ estimation in favor of the AHL-network relative to two US-based detectors is also nominal - at about 25\%. As the source distance increases resulting in a decrease in SNR, the improvement in precision for $D_{L}$ and $\eta$ does not change much for comparable SNRs in the three detectors. However, we find that for low SNR events the precision in $\widetilde{\Lambda}$ improves significantly. For the set of BNS observation at $D_{L}=100 \mathrm{Mpc}$, we find that improvements can be in the range of $20 \%$ to $45 \%$. For the more distant sources, e.g., at $D_{L}=250$ the improvements are generally more than $40 \%$, and can be as high as $70 \%$ in favor of AHL relative to the HL-network. Moreover, for such distant sources, the lack of precision in $\widetilde{\Lambda}$ can render it difficult to rule out the BBH-case corresponding to $\widetilde{\Lambda}=0$, particularly for the soft (SLy4-like) EOS. (As a comparison to the range of $\widetilde{\Lambda}$ parameter for different theoretically motivated neutron star EOS models please refer to [58].) Thus, for the events with relatively weak signals - which will be at farther distances and, hence, in relatively abundant numbers - the source classification (i.e., $\mathrm{BBH}$ vs $\mathrm{BNS} / \mathrm{NSBH}$ ) will get significantly enhanced. This will be important for generating alerts for the subsequent follow-up with astronomical observations across the electromagnetic spectrum.

\section{Sky localization and early warning}

One of the main advantages of expanding the HL network to include LIGO-Aundha is that it substantially improves the localization of CBCs in the sky $[6,37]$. BNSs and a fraction of NSBHs have long been expected to produce prompt counterparts and afterglows in all electromagnetic (EM) bands. For BNS mergers in particular, it has been hypothesized that the post-merger central engine can launch short gamma-ray bursts (sGRBs) [59, 60], kilonovae [61, 62], and radio waves and X-rays before and after merger [63, 64, 65, 66].

These emissions carry information about both the progenitors - e.g., the equation of state of neutron stars - and the circum-merger environment. The prompt, and often, transient emission on the one hand and the late-time afterglow on the other hand complement each other in conveying that information, as was demonstrated amply by the multi-messenger observations of the binary neutron star event, GW170817 [67]. The joint observation of GWs followed by the sGRB, GRB 170817A, and the kilonova AT 2017gfo, [67] confirmed the several-decade-old hypothesis that compact object mergers were progenitors of these exotic transients. However, GW170817 is so far the only gravitational-wave event to be observed in other channels. Improvements in 
GW detectors and expansion of the GW network is therefore required to realize more multi-messenger observations and expand our knowledge about the physical processes that occur in these systems.

The chances of telescopes spotting that EM emission improve if the localization area in the sky associated with the GW signal is small. This is particularly true for tracking down optical counterparts since the fields of view (FOV), or beam sizes, of these telescopes are small (sub-arcminutes) compared to the the typical GW sky-localization area. The small localization with the rapid search strategies [68, 69, 70] can enhance the probability of finding the optical counterpart of the GW source. For prompt and transient emission, a narrow sky-area implies a small number of telescope slews and a quicker locking on to the target before it fades [71]. The search for kilonovae and prolonged afterglows is aided by narrow sky-areas since they are scannable quickly by telescopes and make multiple observations of the same telescope fields of view in those areas more feasible. This, in turn, improves the probablity for spotting their onset. In the case of larger localizations, the early observations are likely to be missed. In some cases (e.g., GW190425 [72]), large localizations can prohibit identification of the EM counterpart entirely. Also, radio follow-up affords complementary observations for day-time and dust-obscured events, where the hunt in optical is difficult. In that case, a small volume in 3D localization is important for the galaxy targeted radio observation to get arcsecond localization [73].

Moreover, if the sky-localization is sharper, then spectroscopy becomes possible, which can provide not only clues on the progenitor composition but also the redshift of the event. Spectroscopy requires longer exposure times. A narrow sky error region implies a smaller number of fields of view to search in for finding the counterpart. This allows for a quicker homing in on potential counterparts and, therefore, extended exposures thereafter. The first discovery of the optical-counterpart of the event GW170817 was after 11 hours of the GW trigger. Detection of the EM-counterparts must be much quicker if their prompt emissions are the desired target.

To demonstrate the benefit of including LIGO-Aundha in the GW network, we simulate a population of binary neutron stars and compare the distribution of GW localization in the two detector networks: HL and AHL. We generate a population of 9,308,544 simulated BNS signals using the TaylorF2 [74, 75, 76, 77] waveform model. Both source-frame component masses are drawn from a Gaussian distribution between $1.0 M_{\odot}<m_{1}, m_{2}<2.0 M_{\odot}$ with mean mass of $1.33 M_{\odot}$ and standard deviation of $0.09 M_{\odot}$, modeled after observations of galactic BNSs [78] (note, however [72]). The component spins are aligned or anti-aligned with respect to the orbital angular momentum with the dimensionless spin amplitude on the neutron stars restricted to 0.05 , motivated by the low spins of BNSs expected to merge within a Hubble time [79, 80]. The signals are distributed uniformly in sky, orientation, and comoving volume up to a redshift of $z=0.4$. We simulate the GW signal and calculate the expected SNR in Gaussian noise considering the three LIGO detectors at A+ sensitivity for each BNS. We mimic the results from a matched-filter GW search pipeline (current low- 
latency matched-filter searches running on LIGO-Virgo data include GstLAL [81, 82], PyCBCLive [83], MBTAOnline [84], SPIIR [85]) by considering the signals that pass a network SNR threshold of 12.0 to be 'detected'. We then calculate the skylocalization posteriors for the detected candidates using a rapid Bayesian localization tool, BAYESTAR [86]. We use the most recent BNS local merger rate from [72] of $320_{-240}^{+410} \mathrm{Gpc}^{-3} \mathrm{yr}^{-1}$ to estimate the number of events detected per year in the detector network.

In Figure 4, we show the distributions (left: cumulative, right: density) of the sky localizations (90\% credible interval) of the BNSs that pass the fiducial SNR threshold of 12 for the two detector networks: HL in purple and AHL in blue. The shaded regions show the uncertainty in the number of detections due to the uncertainty in the current local BNS merger rate of $320 \mathrm{Gpc}^{-3} \mathrm{yr}^{-1}$ [72]. The improvement due to the addition of LIGO-Aundha to the network is clearly visible in this figure; the AHL network detects about twice $(17-175)$ as many signals as the HL $(8-84)$ network. Further the peak of distribution for HL is around $800 \mathrm{deg}^{2}$, about twice that for the AHL network.

Figure 5 shows the shape and the areal projection of several localizations on the sky. The HL (AHL) localizations are shown in purple (blue) contours. Most of the HL localizations are long arcs that extend over both hemispheres. When we include LIGO-Aundha, the degeneracy breaks and the localizations typically shrink to one of the hemispheres. In this plot, a few blue contours have no corresponding purple contours. These are the events detected by AHL but not by HL. The orientations of the HL localizations are concentric. On the other hand AHL localizations are randomly oriented. This is because a single baseline offers a single time delay for any event, which in turn is consistent with source sky-positions that all lie on a single circle in the sky. Of course, detector antenna functions help localize the source position further in those circles, thereby, reducing the localization area to arcs. The addition of a third detector provides additional time delays that aid in reducing the error patches further, as evident in the blue error contours.

The study in Figure 6 compares gravitational-wave sky-localization by HL and AHL for similar sources in different parts of the sky. For this purpose, we divide the entire sky into equal-area pixels in HEALPix * format of NSIDE 16 [87]. We inject one BNS source in each of the 3072 pixels and calculate the localization area of the source using BAYESTAR for HL and AHL. All the injected sources are of $m_{1}, m_{2}=1.4 M_{\odot}$ at a distance of $100 \mathrm{Mpc}$ and have an orbital inclination of $5 \mathrm{deg}$. In Figure 6, the colorbar represents the area of the $90 \%$ credible region of the localization in $\mathrm{deg}^{2}$. In other words, the pixel value is the $90 \%$ probable area of the localization of the source injected in that pixel. For the HL network, the smallest localization area is $\sim 45 \mathrm{deg}^{2}$, and the largest one is $\sim 1732 \mathrm{deg}^{2}$. On the other hand, the smallest and the largest localization areas for AHL are $\sim 1 \mathrm{deg}^{2}$ and $\sim 21 \mathrm{deg}^{2}$. Compared to HL, the AHL sky areas are

* A HEALPix map parameterized by the variable NSIDE is a representation of the full-sky filled with $N_{p i x}=12 \times N S I D E^{2}$ equal-area pixels. The value corresponding to a pixel is the probability of finding the GW source in that pixel in the sky. 

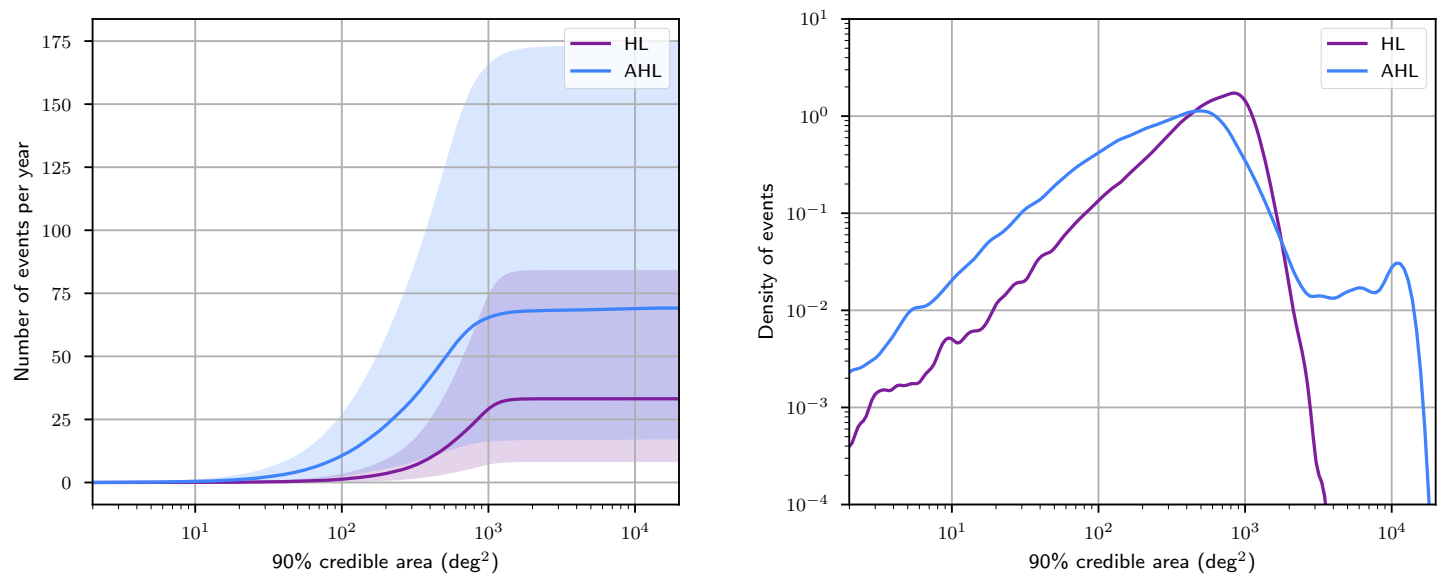

Figure 4: Distributions (left: cumulative, right: density) of the sky localizations (90\% credible interval) of the BNSs that pass the fiducial SNR threshold of 12 for the two detector networks: HL (purple) and AHL (blue). Using the latest median BNS merger rate from [72] of $320 \mathrm{Gpc}^{-3} \mathrm{yr}^{-1}$, we find that the HL (AHL) network is expected to detect $\sim 33$ (69) events per year. The shaded regions represent the uncertainty in the BNS merger rate estimate.

not smaller by a constant factor. How much the area shrinks depends on the true position of the source. The results in Figure 5 and Figure 6 are consistent and are two different representations of sky-localization analysis using the same aforementioned code. Furthermore, in Figure 6 we observe that the AHL network localizes all the 3072 events with $90 \%$ credible area within 20 sq.deg. On the other hand, the HL localizes 793 events within $100 \mathrm{deg}^{2}$ and 239 events within $50 \mathrm{deg}^{2}$.

\subsection{Early warning of binary neutron star mergers}

August 17, 2017 saw the beginning of a new era in multi-messenger astronomy. The joint detection of GWs by the LIGO and Virgo interferometers and the sGRB by the Fermi-GBM and INTEGRAL satellite from the BNS coalescence, GW170817 [51, 67] confirmed the long-standing hypothesis that compact object mergers were progenitors of short GRBs. Apart from the gamma-ray burst, which was observed $\sim 2 \mathrm{~s}$ after the merger event, the first manual follow-up observations took place $\sim 8$ hours after the epoch of merger [67]. This delay was caused by the delay in sending out GW information: the GW alert was sent out $\sim 40$ minutes [88], and the sky localization 4.5 hours [89] after the signal arrived on earth. By the time EM telescopes participating in the follow-up program received the alerts the source was below the horizon for them.

For a fraction of BNS events it will be possible to issue alerts up to $\delta t \sim 60 \mathrm{~s}$ before the epoch of merger [90, 91, 92]. Pre-merger or early warning detections will facilitate electromagnetic observations of the prompt emission, which encodes the initial conditions of the outflow and the state of the merger remnant. Early 


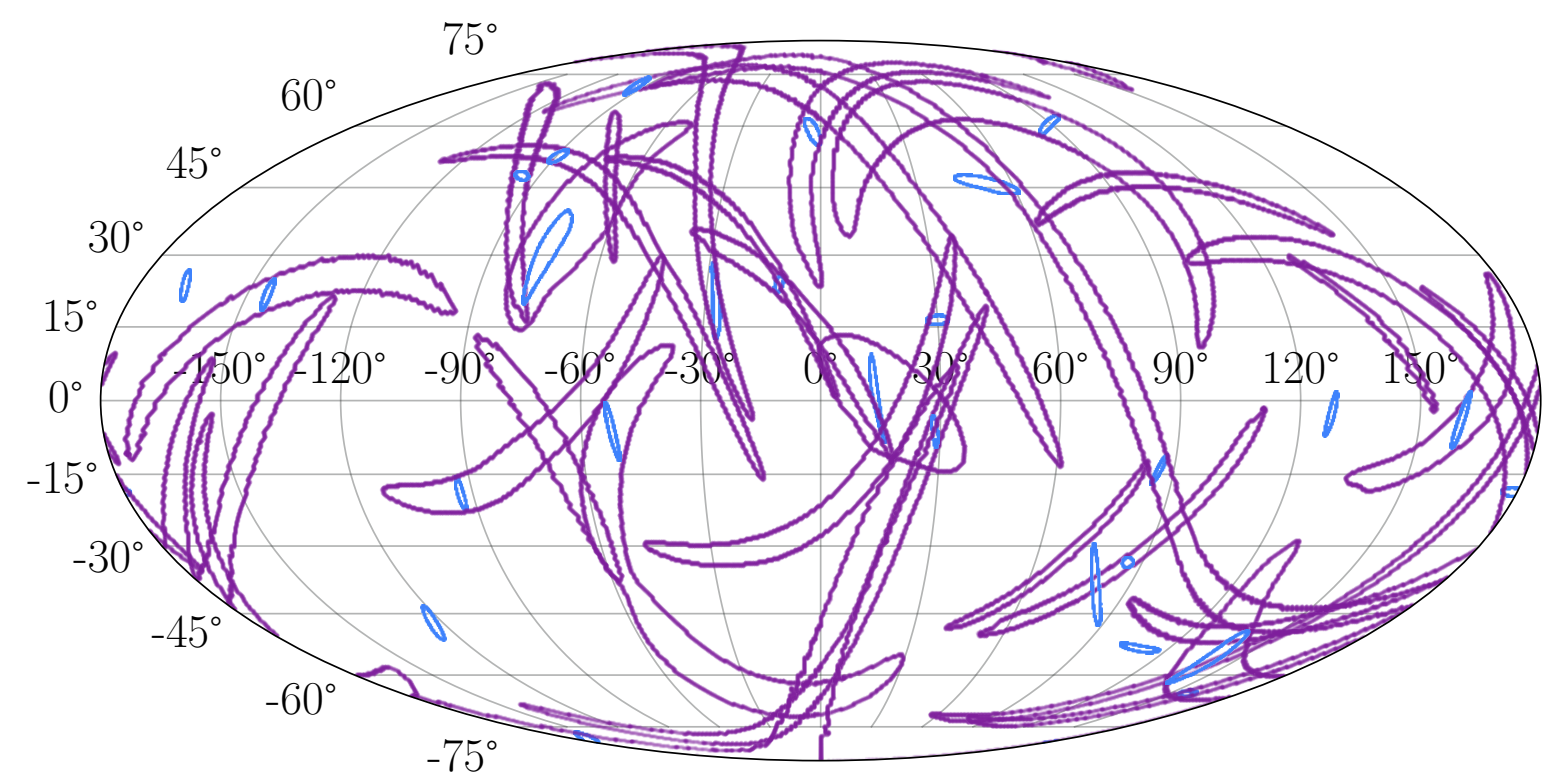

Figure 5: Shown above are the $90 \%$ probability contours of source localization on the sky. The blue and purple contours correspond to the networks AHL and HL, respectively, for the same sources selected from our BNS simulations, and exhibit the improvement achieved due to the inclusion of the third detector in LGN. Note that AHL detects every signal that is detected by HL. The isolated blue contours correspond to sources detected by AHL but not by HL.

optical and ultraviolet observations will be key to our understanding of $r$-process nucleosynthesis [93] and shock-heated ejecta [94], while prompt X-ray emission would reveal the final state of the remnant [95, 96, 97]. Early observations made in the radio band could indicate pre-merger magnetosphere interactions [98], and might be able to test models that predict BNS mergers as a possible precursor of fast radio bursts [99, 100, 101]. Early-warning GW alerts have recently been implemented [102, 103] and also demonstrated [104] recently.

Here we will compare the prospects of early-warning detection of GWs from BNSs for the two detector networks: HL and AHL. We follow the framework laid out in [102], which implemented an early warning GW pipeline using the GstLAL matched-filtering software suite [105]. In particular, we consider 6 different discrete frequency cutoffs $29 \mathrm{~Hz}, 32 \mathrm{~Hz}, 38 \mathrm{~Hz}, 49 \mathrm{~Hz}, 56 \mathrm{~Hz}$, and $1024 \mathrm{~Hz}$ to analyze signal recovery at (approximately) $58 \mathrm{~s}, 44 \mathrm{~s}, 28 \mathrm{~s}, 14 \mathrm{~s}, 10 \mathrm{~s}$, and $0 \mathrm{~s}$ before merger. We use the population of BNSs described earlier and the same criteria for 'detected' signal, the signals that pass an SNR threshold of 12.0 in each frequency configuration are detected with the corresponding pre-merger latency. We then use BAYESTAR to localize all the detected signals for each frequency configuration.

Our results are shown in Figure 7. For both networks (left: HL, right: AHL), we show the cumulative distributions of the $90 \%$ credible intervals of the sky localizations 

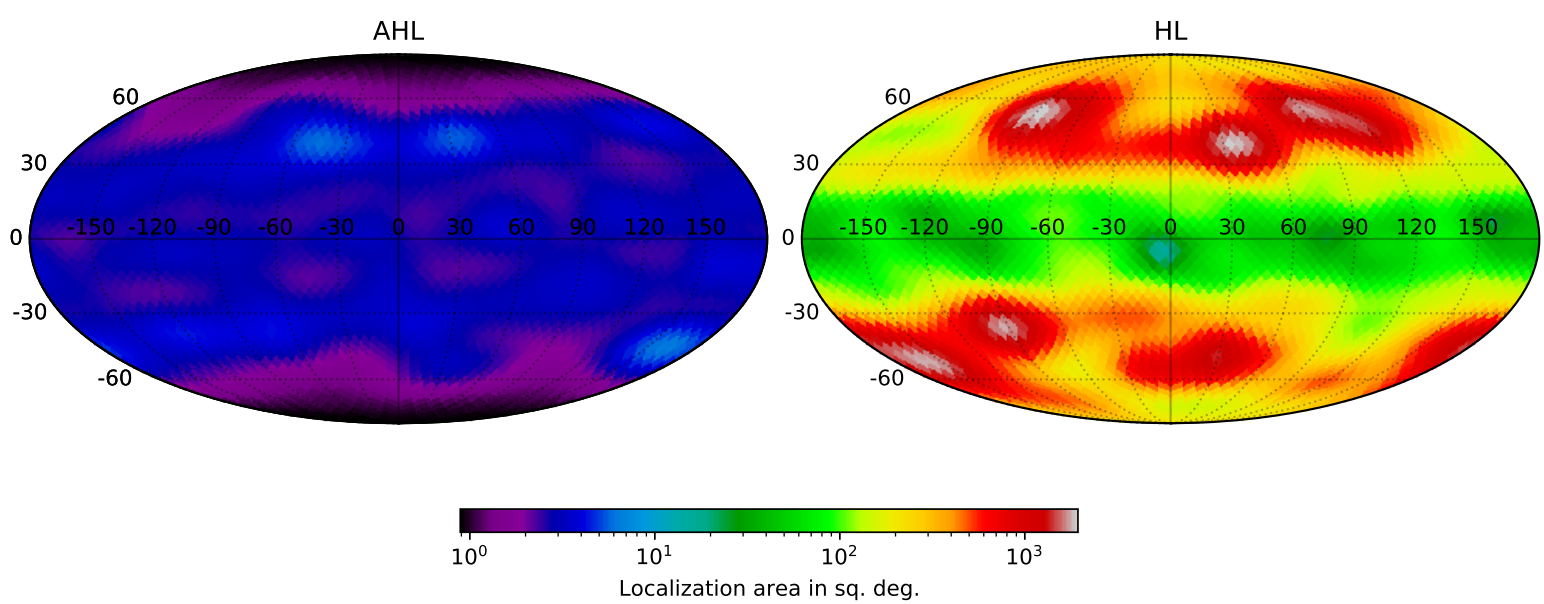

Figure 6: Localization area for BNS sources across the sky for the AHL and HL networks. Typically, smaller areas for AHL compared to HL show the degree of improvement the presence of the third LIGO detector in the LGN brings about. The sky map is divided into equal-area pixels in the HEALPix format. Each pixel has one injected BNS source in it and the colorbar value of the pixel is the $90 \%$ probable area of the localization of the source calculated by BAYESTAR. All the BNSs are injected at 100 Mpc, and have $m 1=m 2=1.4 M_{\odot}$ and $5^{\circ}$ inclination.

for each pre-merger time considered in our simulation. The $y$-axis is translated to number of detections per year assuming the current median BNS merger rate estimate and $100 \%$ duty cycle of the networks. We note that at each frequency and pre-merger time configuration, the AHL network is able to detect about twice the number of events as compared to the HL network. In particular, the number of events per year that could be detected at least $10 \mathrm{~s}$ before merger is 7 (15) for the HL (AHL) network. Adding LIGO-Aundha to the network will also greatly reduce the area in the sky to which these events can be localized, thereby vastly improving prospects of observing EM emissions before and/or at merger. The HL network is expected to detect one event every two years before merger that is also localized to $1000 \mathrm{deg}^{2}$, while the AHL network is expected to detect $\sim 5$ events every year before merger that are also localized to $1000 \mathrm{deg}^{2}$.

These localizations are quite large for optical telescopes, which generally have very small FOVs in comparison. With the current estimates of the BNS merger rate, the AHL network can detect 1 event every $\sim 4$ years $15 \mathrm{~s}$ before merger localized to $100 \mathrm{deg}^{2}$. For the HL network, such an event will be detected once every $\sim 85$ years.

Some of the largest field telesopes, such as the BlackGEM array $\left(0.65 \mathrm{~m} / 2.7 \mathrm{deg}^{2}\right.$ per telescope) with 3 telescopes planned in the first phase of operation eventually expanding to 15 telescopes [106], the Zwicky Transient Facility $\left(1.2 \mathrm{~m} / 47 \mathrm{deg}^{2}\right)$ [107], the Dark Energy Camera (4m/3.8 $\left.\mathrm{deg}^{2}\right)$ [108], the Rubin Observatory (8.4 m/9.6 deg ${ }^{2}$ ) [109], 

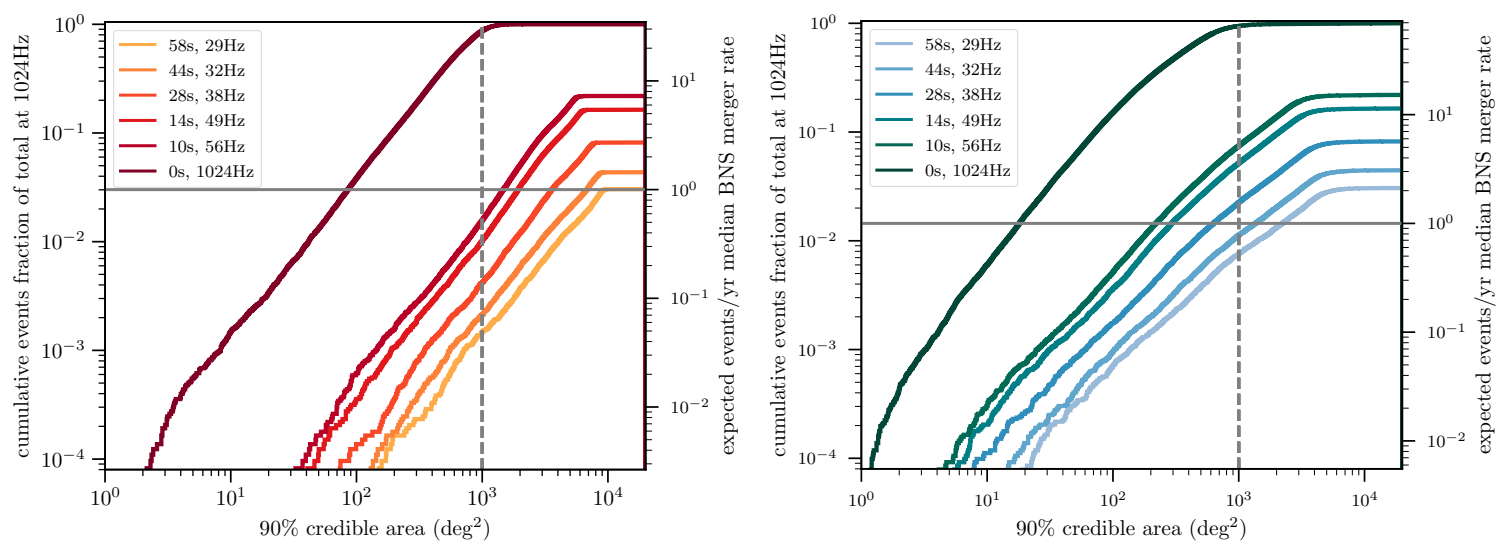

Figure 7: Cumulative distributions of the sky localizations (90\% credible interval) of the BNSs that pass the fiducial SNR threshold of 12 for the two detector networks: HL (left) and AHL (right). The different colors show the different frequency bandwidths or pre-merger times considered in our work. Using the latest median BNS merger rate from [72] of $320 \mathrm{Gpc}^{-3} \mathrm{yr}^{-1}$, we find that the HL (AHL) network is expected to detect $\sim 7$ (15) mergers per year at least $10 \mathrm{~s}$ before merger. Out of these, the HL network is expected to detect one event every two years before merger that is also localized to $1000 \mathrm{deg}^{2}$, while the AHL network is expected to detect $\sim 5$ events every year before merger that are also localized to $1000 \mathrm{deg}^{2}$.

the Swope Telescope $\left(1 \mathrm{~m} / 7 \mathrm{deg}^{2}\right)$ [110], the Subaru Telescope $\left(8.2 \mathrm{~m} / 1.7 \mathrm{deg}^{2}\right)$ [111], etc. all have FOVs only a small fraction of the GW localizations. Therefore, adding LIGOAundha to the LGN will enhance the chances of observing any pre-merger and prompt emissions. Similarly, a larger network involving LGN as well as non-LGN detectors is expected to boost these chances further.

\section{Tests of GR}

\subsection{Improved constraints on deviations from GR}

Most of the tests of general relativity performed in [112, 113, 114] are null tests in the sense that they look for deviations around the expectation from GR. These deviation parameters can be measured from each detected event and, assuming certain properties, can be combined across multiple events to get tighter constraints. Measurement of deviations in the post-Newtonian parameters [115, 116, 117, 118], inspiral-merger-ringdown consistency test [119, 120, 121], and the upper bound on the mass of the graviton [122], etc., fall under this category.

We illustrate the potential of getting tighter bounds on the GR deviation parameters with the AHL network using the measurement of the mass of the graviton. In GR, GWs are non-dispersive. Hence the corresponding force carrier, graviton, should have zero rest mass. But there are alternative theories to GR that permit 
a non-trivial dispersion relation. We assume the following phenomenological form [123, 122, 124, 125, 126, 127, 128, 129],

$$
E^{2}=p^{2} c^{2}+A_{\alpha} p^{\alpha} c^{\alpha}
$$

where $E$ and $p$ are the energy and momentum of the GW, and $A_{\alpha}$ and $\alpha$ are phenomenological parameters. The phenomenological parameters are related to the graviton's mass by $m_{g}=\sqrt{A_{0}} / c^{2}$, with the condition $A_{0}>0$.

The 90\% upper bound on the mass of the graviton measured from GW150914 is $9.9 \times 10^{-23} \mathrm{eV} / \mathrm{c}^{2}$ [113]. If the graviton is indeed massless, then this constraint will get tighter with more detections, especially, with those that turn out to be louder than GW150914. We assume that the upper bound scales inversely with the SNR of the detection. So, for a given event $i$ with $\operatorname{SNR} \rho_{i}$, the $90 \%$ upper bound on the graviton mass $\sigma_{i}$ is given by $\sigma_{i}=\sigma_{0} \rho_{0} / \rho_{i}$. Here, $\sigma_{0}$ and $\rho_{0}$ are the $90 \%$ upper bound on graviton mass and the SNR obtained from GW150914. If $N$ events are detected, we can combine the individual $\sigma_{i}$ to obtain the following joint constraint:

$$
\sigma_{\mathrm{comb}}=\frac{1}{\sqrt{\sum_{i=1}^{N} \sigma_{i}^{-2}}}=\frac{\sigma_{0} \rho_{0}}{\sqrt{\sum_{i=1}^{N} \rho_{i}^{2}}} .
$$

We simulate sources in the HL and AHL networks using the models specified in Sec. 1.2, and consider only those events that are detected by the multi-detector coincidence criterion (criterion (ii) in Sec. 2). We find that there are, on average, 502 events per year in the HL network and 754 events per year in AHL network that satisfy this criterion. We apply the above prescription to obtain the constraints and find $\sigma_{\text {comb }}^{\mathrm{HLA}} / \sigma_{\text {comb }}^{\mathrm{HL}} \approx 0.8$, i.e. the constraints obtained with the AHL network are $20 \%$ tighter than the ones obtained with the HL network.

\subsection{Constraints on the nature of $G W$ polarisations}

In GR, GWs have only two independent polarisation states - i.e., two transverse quadrupole (or tensor) modes. In comparison, a general metric theory of gravity can admit up to six polarisation modes. In this sense, GW polarisations offer an interesting test of GR. GW polarisations can be constrained from observations of spinning neutron stars [130, 131] and stochastic background [132, 133, 134], as well as from observations of compact binary mergers [135, 136, 137, 138, 139, 140, 141]. While the detectability of spinning neutron stars or stochastic background is uncertain, we are expecting to detect hundreds to thousands of compact binary mergers in the next few years using ground-based GW detectors. Note that each GW detector observes only one linear combination of these polarisations. Due to the near co-alignment of the LIGO-Hanford and -Livingston detectors, they measure essentially the same linear combination of polarisations in a binary merger signal. Hence, currently the LIGO detectors alone are practically incapable of resolving even the two polarisation states predicted by GR. Additional detectors around the globe, including LIGO-Aundha, will enable observing those two states and potentially constrain the additional non-GR polarisation states. 
Given the data from a network of GW detectors, we can compare the posterior probabilities of different hypotheses, for example, one hypothesis stating that the binary phase evolution is exactly as predicted by GR, while the alternative hypothesis accommodating the presence of additional modes [135]. Motivated by the limited number of linearly independent detectors to observe the polarisation modes, the current probes of the nature of GW polarisations have employed highly simplified hypotheses as alternatives to GR. That is, the alternative hypothesis assumes that the polarisations contain only scalar modes $\left(h_{\mathrm{b}}\right.$ and $\left.h_{\mathrm{l}}\right)$ or only vector modes $\left(h_{\mathrm{x}}\right.$ and $\left.h_{\mathrm{y}}\right)$ or only tensor modes $\left(h_{+}\right.$and $\left.h_{\times}\right)[138,142,137,140]$.

We perform a simulation study that compares the ability of the 3-detector network involving LIGO-Aundha to distinguish different polarisation models, as compared to the 2-detector network involving only LIGO Hanford and Livingston. For each polarisation hypothesis - tensor $H_{t}$, vector $H_{v}$, and scalar $H_{s}$, the model waveforms are generated using the corresponding antenna patterns, but always assuming that the time evolution of the polarisations follows that of the GR modes. That is, $h_{\mathrm{b}}(t)=$ $h_{\mathrm{x}}(t)=h_{+}(t)$ and $h_{\mathrm{l}}(t)=h_{\mathrm{y}}(t)=h_{\times}(t)$. Since each detector $I$ has different antenna pattern functions $F_{I}^{A}$ for each GW polarisation $A$, the strain measured is a different linear combination of the polarisation modes: $h_{I}(t)=F_{I}^{A}(\alpha, \delta, \psi, t) h_{A}(t)$. For simplicity, no noise is added to the simulated signals. Further, we considered GW signals from non-spinning binary black holes as our signal model.

We use the standard Gaussian likelihood model for estimating the posteriors of the parameters under different polarisation hypotheses (see, e.g., [143]), using the BILBY software package [36]. Posteriors are computed over the parameters $\left(m_{1}, m_{2}, \alpha, \delta, d_{L}, \iota, \psi, \phi_{0}, t_{0}\right)$, where $t_{0}$ and $\phi_{0}$ are the arrival time and phase, respectively. We use uniform priors in redshifted component masses of the binary $\left(m_{1}, m_{2} \in[3,500] M_{\odot}\right)$, isotropic priors in sky location (uniform in $\alpha$, $\sin \delta$ ) and orientation (uniform in $\cos \iota, \phi_{0}$ ), uniform prior in polarisation angle $\psi$, and a volumetric prior $\propto d_{L}^{2}$ on luminosity distance. The Bayesian evidence of each polarisation model is obtained as part of the parameter estimation.

We simulate $200 \mathrm{GR}$ (tensor) double coincident injections for each one of HL and AHL networks, and do parameter estimation and compute evidences $P\left(d \mid H_{p}\right)$ for each of the polarisation hypothesis $H_{p} \in\left\{H_{t}, H_{v}, H_{s}\right\}$. From those evidences, the likelihood ratio (Bayes factor) for tensor $v s$ vector $\left(B_{v}^{t}\right)$ and tensor $v s$ scalar $\left(B_{s}^{t}\right)$ hypotheses are calculated for various combinations of detectors. The distributions of the Bayes factors $B_{s}^{t}:=P\left(d \mid H_{t}\right) / P\left(d \mid H_{s}\right)$ and $B_{v}^{t}=P\left(d \mid H_{t}\right) / P\left(d \mid H_{v}\right)$ are plotted in Fig. 8. We can see that the 3-detector AHL network has a much better ability (larger Bayes factors) to distinguish the polarisation models as compared to the 2-detector HL network.

\section{Conclusions and summary}

We have analyzed the performance of the LIGO Global Network, in particular focusing on the improvement that comes about from the addition of a LIGO detector in India. 


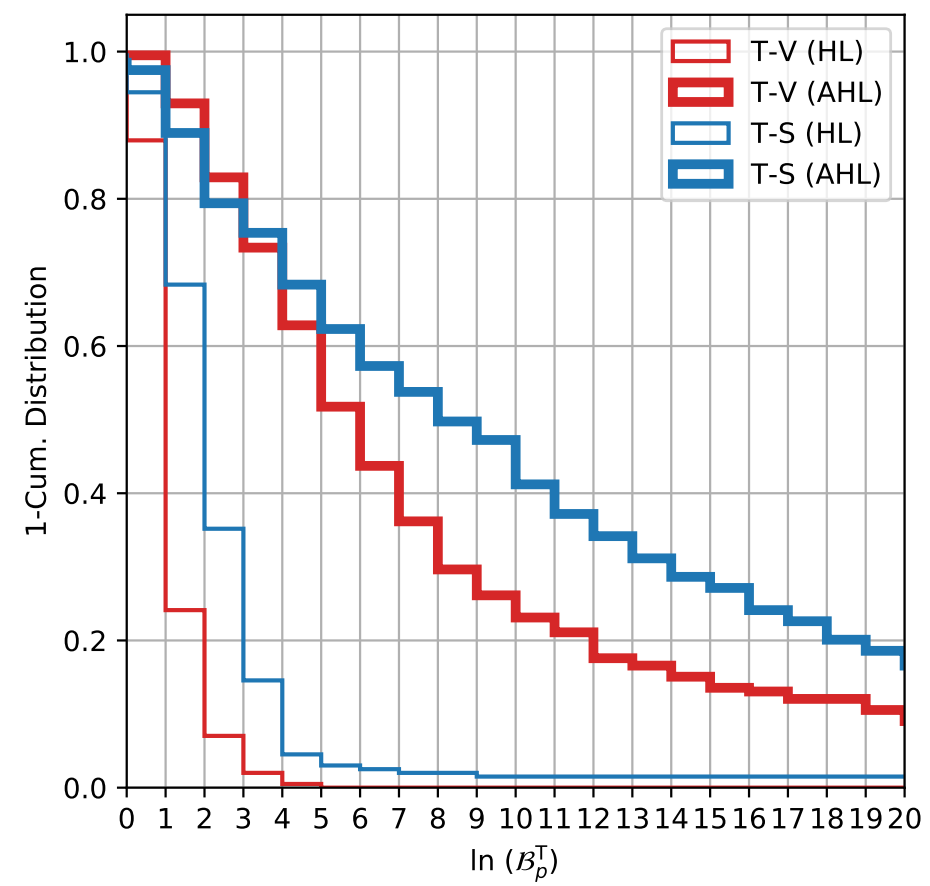

Figure 8: The distribution of the Bayes factors, $B_{v}^{t}(\mathrm{red})$ and $B_{s}^{t}(\mathrm{blue})$, that show our ability to distinguish different polarisation models. The simulated signals always follow tensor polarisations (as predicted by GR) and Bayesian evidence of three different polarisation models are computed. We can see that the 3-detector AHL network has a much better ability (larger Bayes factors) to distinguish the polarisation models as compared to the 2-detector HL network(thin).

We focused on compact binary coalescences, involving black holes and neutron stars, as our sources. We find, overall, a significant improvement in the precision with which various binary parameters can be measured. This is especially significant for the sky localization of compact binaries as well as the related ability to issue early warning for BNS mergers. Precise localization is crucial in spotting and associating kilonovae with such mergers. Correct associations are necessary for understanding the influence of progenitor properties on kilonova properties, such as their spectra. They also have a bearing on constraining neutron star equation of state and the measurement of the Hubble parameter without invoking the cosmic-distance ladder.

Reducing the alert time for these mergers by several to a few tens of seconds can impact the ability of astronomers to slew their telescopes in time to capture prompt afterglow emissions, not to mention pre-merger EM signals. Prompt afterglows were missed in the observation of GW170817 and can provide important clues about the short GRB engine. This is one of the next frontiers in GRB physics that the GW network can contribute to. 
The increased detection rate of CBCs with the addition of the third LIGO detector in India will also allow stronger constraints to be placed on possible deviations from GR. By assuming that constraints from a given signal would scale inversely with the SNR, and combining constraints across events, we find that the AHL network will offer a $20 \%$ improvement over the HL network on the graviton mass upper limit. We also showed how an additional detector in the network aids in discriminating among different polarisation models: Here we limited ourselves to models where GWs have only tensor, only vector or only scalar polarisation modes - taking all modes to have the same phase evolution; some of these assumptions can be relaxed in the future by using the null stream reconstruction [144, 139, 141].

While our primary focus here has been CBCs, the LIGO Global Network will also impact science pursuable with other signals. One such signal is an astrophysical stochastic gravitational-wave background (SGWB) arising from the superposition of inspiral signals from populations of binaries of black holes and neutron stars [145, 146]. By combining the detections of dozens of individual binaries, on the one hand, with upper-limits on the power spectra of SGWB, on the other hand, past studies [147, 148] have constrained the rate of evolution of CBCs over redshift. The spread in this rate will shrink by $20 \%$ with the third detector added to this network. The addition of a third detector will also help to understand the correlated terrestrial noise sources, which in turn will play a crucial role in confidently claiming any SGWB detection. Moreover, if the astrophysical SGWB has significant anisotropies, probing them requires better sky coverage. LIGO-India's inclusion in the existing detector network will help to resolve these finer angular structures using the existing mapping techniques [149, 150, 151, 152].

In the future, it will be interesting to study other types of sources (and not just compact binaries), the effects of realistic interferometer noise, and the presence of other detectors in the network.

\section{Acknowledgements}

We would like to thank our colleagues in the LIGO-India Scientific Collaboration and the LIGO-India Project for valuable inputs. We appreciate the several discussions we had with members of the various working groups in the LIGO-Virgo-KAGRA collaborations. In particular, we thank K. G. Arun, Bala Iyer, Shivaraj Kandhasamy, Jose Matthew, Fred Raab, Rory Smith, and Tarun Souradeep for valuable discussions and inputs. This work makes use of NUMPy [153], ScIPy [154], MATPLOTLIB [155], AStroPy [156, 157], JUPYTER [158], Dynesty [159], BILby [36] and PESummary [160] software packages. Thanks are also due to the Department of Science and Technology (DST) and the Department of Atomic Energy (DAE) of India. Specifically, MS acknowledges the support from the Infosys Foundation, the Swarnajayanti fellowship grant DST/SJF/PSA-01/2017-18, and the support from the National Science Foundation with grants PHY-1806630 and PHY-2010970, AP acknowledges support 
from the DST-SERB Matrics grant MTR/2019/001096 and SERB-Power-fellowship grant SPF/2021/000036, DST,India. AM acknowledges support from the DST-SERB Start-up Research Grant SRG/2020/001290, and PA, AV, and SG acknowledge support from DAE under project no. RTI4001. PA's research was also supported by the Max Planck Society through a Max Planck Partner Group at ICTS-TIFR and by the Canadian Institute for Advanced Research through the CIFAR Azrieli Global Scholars program. SS is supported by an Eberly postdoctoral fellowship at Pennsylvania State University and B.S.S. is supported by NSF grants PHYS-1836779, PHYS-2012083 and AST-2006384. Thanks are due to computational support provided by the Alice (ICTSTIFR) and Sarathi (IUCAA) clusters and computing resources in SINP. In addition, the authors are also grateful for the computational resources provided by LIGO Laboratory and Leonard E Parker Center for Gravitation, Cosmology and Astrophysics at the University of Wisconsin-Milwaukee and supported by National Science Foundation Grants PHY-0757058, PHY-0823459, PHY-1626190 and PHY-1700765. This paper has been assigned the internal LIGO preprint number P2100073. 
[1] Aasi J et al. (LIGO Scientific Collaboration) 2015 Class. Quantum Grav. 32074001 (Preprint 1411.4547)

[2] Acernese F et al. (Virgo Collaboration) 2015 Class. Quantum Grav. 32024001 (Preprint 1408. 3978)

[3] Abbott R et al. (LIGO Scientific, Virgo) 2020 (Preprint 2010.14527)

[4] KAGRA Collaboration, Akutsu T, Ando M, Arai K, Arai Y, Araki S, Araya A, Aritomi N, Asada H, Aso Y, Bae S, Bae Y, Baiotti L, Bajpai R, Barton M A, Cannon K, Cao Z, Capocasa E, Chan M, Chen C, Chen K, Chen Y, Chiang C Y, Chu H, Chu Y K, Eguchi S, Enomoto Y, Flaminio R, Fujii Y, Fujikawa F, Fukunaga M, Fukushima M, Gao D, Ge G, Ha S, Hagiwara A, Haino S, Han W B, Hasegawa K, Hattori K, Hayakawa H, Hayama K, Himemoto Y, Hiranuma Y, Hirata N, Hirose E, Hong Z, Hsieh B H, Huang C Z, Huang H Y, Huang P, Huang Y C, Huang Y, Hui D C Y, Ide S, Ikenoue B, Imam S, Inayoshi K, Inoue Y, Ioka K, Ito K, Itoh Y, Izumi K, Jeon C, Jin H B, Jung K, Jung P, Kaihotsu K, Kajita T, Kakizaki M, Kamiizumi M, Kanda N, Kang G, Kashiyama K, Kawaguchi K, Kawai N, Kawasaki T, Kim C, Kim J, Kim J C, Kim W S, Kim Y M, Kimura N, Kita N, Kitazawa H, Kojima Y, Kokeyama K, Komori K, Kong A K H, Kotake K, Kozakai C, Kozu R, Kumar R, Kume J, Kuo C, Kuo H S, Kuromiya Y, Kuroyanagi S, Kusayanagi K, Kwak K, Lee H K, Lee H W, Lee R, Leonardi M, Li T G F, Li K L, Lin L C C, Lin C Y, Lin F K, Lin F L, Lin H L, Liu G C, Luo L W, Majorana E, Marchio M, Michimura Y, Mio N, Miyakawa O, Miyamoto A, Miyazaki Y, Miyo K, Miyoki S, Mori Y, Morisaki S, Moriwaki Y, Nagano K, Nagano S, Nakamura K, Nakano H, Nakano M, Nakashima R, Nakayama Y, Narikawa T, Naticchioni L, Negishi R, Nguyen Quynh L, Ni W T, Nishizawa A, Nozaki S, Obuchi Y, Ogaki W, Oh J J, Oh K, Oh S H, Ohashi M, Ohishi N, Ohkawa M, Ohta H, Okutani Y, Okutomi K, Oohara K, Ooi C P, Oshino S, Otabe S, Pan K, Pang H, Parisi A, Park J, Pena Arellano F E, Pinto I, Sago N, Saito S, Saito Y, Sakai K, Sakai Y, Sakuno Y, Sato S, Sato T, Sawada T, Sekiguchi T, Sekiguchi Y, Shao L, Shibagaki S, Shimizu R, Shimoda T, Shimode K, Shinkai H, Shishido T, Shoda A, Somiya K, Son E J, Sotani H, Sugimoto R, Suresh J, Suzuki T, Suzuki T, Tagoshi H, Takahashi H, Takahashi R, Takamori A, Takano S, Takeda H, Takeda M, Tanaka H, Tanaka K, Tanaka K, Tanaka T, Tanaka T, Tanioka S, Tapia San Martin E N, Telada S, Tomaru T, Tomigami Y, Tomura T, Travasso F, Trozzo L, Tsang T, Tsao J S, Tsubono K, Tsuchida S, Tsuna D, Tsutsui T, Tsuzuki T, Tuyenbayev D, Uchikata N, Uchiyama T, Ueda A, Uehara T, Ueno K, Ueshima G, Uraguchi F, Ushiba T, van Putten M H P M, Vocca H, Wang J, Washimi T, Wu C, Wu H, Wu S, Xu W R, Yamada T, Yamamoto K, Yamamoto K, Yamamoto T, Yamashita K, Yamazaki R, Yang Y, Yokogawa K, Yokoyama J, Yokozawa T, Yoshioka T, Yuzurihara H, Zeidler S, Zhan M, Zhang H, Zhao Y and Zhu Z H 2020 arXiv e-prints arXiv:2008.02921 (Preprint 2008.02921)

[5] Iyer B et al. 2011 LIGO-India, proposal of the consortium for Indian initiative in gravitationalwave observations (IndIGO) URL https://dcc.ligo.org/LIGO-M1100296/public

[6] Fairhurst S 2014 Journal of Physics: Conference Series 484012007 (Preprint 1205.6611)

[7] Miller J, Barsotti L, Vitale S, Fritschel P, Evans M and Sigg D 2015 Phys. Rev. D 91(6) 062005 URL https://link.aps.org/doi/10.1103/PhysRevD.91.062005

[8] Abbott R et al. (LIGO Scientific, Virgo) 2020 (Preprint 2010.14533)

[9] Kalogera V 2000 Astrophys. J. 541 319-328 (Preprint astro-ph/9911417)

[10] Rodriguez C L, Zevin M, Pankow C, Kalogera V and Rasio F A 2016 Astrophys. J. Lett. 832 L2 (Preprint 1609.05916)

[11] Ade P A R et al. (Planck) 2016 Astron. Astrophys. 594 A13 (Preprint 1502.01589)

[12] Abbott B P et al. (LIGO Scientific, Virgo) 2019 Phys. Rev. X 9031040 (Preprint 1811.12907)

[13] Abbott B et al. (LIGO Scientific, Virgo) 2019 Astrophys. J. Lett. 882 L24 (Preprint 1811.12940)

[14] Abbott B P et al. (LIGO Scientific, Virgo) 2020 Class. Quant. Grav. 37055002 (Preprint 1908. 11170)

[15] Davis D et al. (LIGO) 2021 Class. Quant. Grav. 38135014 (Preprint 2101.11673)

[16] Bose S, Hall B, Mazumder N, Dhurandhar S, Gupta A and Lundgren A 2016 J. Phys. Conf. Ser. 716 012007 (Preprint 1602.02621)

[17] Bose S, Dhurandhar S, Gupta A and Lundgren A 2016 Phys. Rev. D 94122004 (Preprint 
1606.06096)

[18] Finn L S 2001 Phys. Rev. D 63102001 (Preprint gr-qc/0010033)

[19] Pai A, Dhurandhar S and Bose S 2001 Phys. Rev. D64(4) 042004 (Preprint 0009078)

[20] Abbott B et al. (KAGRA, LIGO Scientific, VIRGO) 2018 Living Rev. Rel. 213 (Preprint 1304.0670)

[21] Cutler C and Flanagan E E 1994 Phys. Rev. D 49 2658-2697 (Preprint gr-qc/9402014)

[22] Abbott B P et al. (LIGO Scientific Collaboration and Virgo Collaboration) 2016 Phys. Rev. Lett. 116 061102 (Preprint 1602.03837)

[23] Abbott R et al. (LIGO Scientific, Virgo) 2020 Phys. Rev. Lett. 125101102 (Preprint 2009. 01075)

[24] Ajith P, Hannam M, Husa S, Chen Y, Brügmann B, Dorband N, Müller D, Ohme F, Pollney D, Reisswig C et al. 2011 Physical review letters 106241101

[25] Buonanno A and Damour T 1999 Phys. Rev. D 59084006 (Preprint gr-qc/9811091)

[26] Buonanno A and Damour T 2000 Phys. Rev. D 62064015 (Preprint gr-qc/0001013)

[27] Barausse E and Buonanno A 2010 Phys. Rev. D 81084024 (Preprint 0912.3517)

[28] Field S E, Galley C R, Hesthaven J S, Kaye J and Tiglio M 2014 Phys. Rev. X 4031006 (Preprint 1308.3565)

[29] Blackman J, Field S E, Galley C R, Szilágyi B, Scheel M A, Tiglio M and Hemberger D A 2015 Phys. Rev. Lett. 115121102 (Preprint 1502.07758)

[30] Varma V, Field S E, Scheel M A, Blackman J, Kidder L E and Pfeiffer H P 2019 Phys. Rev. D 99 064045 (Preprint 1812.07865)

[31] Hannam M, Schmidt P, Bohé A, Haegel L, Husa S et al. 2014 Phys.Rev.Lett. 113151101 (Preprint 1308.3271)

[32] Schmidt P, Ohme F and Hannam M 2015 Phys. Rev. D91 024043 (Preprint 1408.1810)

[33] Husa S, Khan S, Hannam M, Pürrer M, Ohme F, Jiménez Forteza X and Bohé A 2016 Phys. Rev. D93 044006 (Preprint 1508.07250)

[34] Khan S, Husa S, Hannam M, Ohme F, Pürrer M, Jiménez Forteza X and Bohé A 2016 Phys. Rev. D93 044007 (Preprint 1508.07253)

[35] Bohé A, Hannam M, Husa S, Ohme F, Puerrer M and Schmidt P 2016 Phenompv2 - technical notes for lal implementation Tech. Rep. LIGO-T1500602 LIGO Project URL https : / dcc . ligo . org/ LIGO-T1500602

[36] Ashton G, Hübner M, Lasky P D, Talbot C, Ackley K, Biscoveanu S, Chu Q, Divakarla A, Easter P J, Goncharov B, Hernandez Vivanco F, Harms J, Lower M E, Meadors G D, Melchor D, Payne E, Pitkin M D, Powell J, Sarin N, Smith R J E and Thrane E 2019 Apjs 24127 (Preprint 1811.02042) URL https://ui .adsabs .harvard .edu/abs/2019ApJS . .241 . . 27A

[37] Pankow C, Rizzo M, Rao K, Berry C P L and Kalogera V 2020 Astrophys. J. 90271 (Preprint 1909.12961)

[38] Ajith P and Bose S 2009 Phys. Rev. D 79084032 (Preprint 0901.4936)

[39] Abbott B P et al. (LIGO Scientific, Virgo) 2021 Astrophys. J. 909218 (Preprint 1908. 06060)

[40] Abbott B P et al. (LIGO Scientific, Virgo, 1M2H, Dark Energy Camera GW-E, DES, DLT40, Las Cumbres Observatory, VINROUGE, MASTER) 2017 Nature 551 85-88 (Preprint 1710.05835)

[41] Schutz B F 1986 Nature 323 310-311

[42] Del Pozzo W 2012 Phys. Rev. D 86043011 (Preprint 1108.1317)

[43] Nair R, Bose S and Saini T D 2018 Phys. Rev. D 98023502 (Preprint 1804.06085)

[44] Chen H Y, Fishbach M and Holz D E 2018 Nature 562 545-547 (Preprint 1712. 06531)

[45] Rodriguez C L, Farr B, Raymond V, Farr W M, Littenberg T B, Fazi D and Kalogera V 2014 Astrophys. J. $\mathbf{7 8 4} 119$ (Preprint 1309.3273)

[46] Arun K G, Tagoshi H, Pai A and Mishra C K 2014 Phys. Rev. D 90024060 (Preprint 1403.6917)

[47] Saleem M 2020 Mon. Not. Roy. Astron. Soc. 493 1633-1639 (Preprint 1905. 00314)

[48] Saleem M, Resmi L, Arun K and Mohan S 2020 The Astrophysical Journal 891130

[49] Hinderer T 2008 The Astrophysical Journal 677 1216-1220 (Preprint 0711.2420)

[50] Wade L, Creighton J D E, Ochsner E, Lackey B D, Farr B F, Littenberg T B and Raymond V 2014 Physical Review D 89103012 (Preprint 1402.5156) 
[51] Abbott B P, Abbott R, Abbott T, Abernathy M, Acernese F, Ackley K, Adams C, Adams T, Addesso P, Adhikari R et al. 2017 Physical review letters 119161101 (Preprint 1710. 05832)

[52] Flanagan É É and Hinderer T 2008 Physical Review D 77021502 (Preprint 0709.1915)

[53] Read J S, Lackey B D, Owen B J and Friedman J L 2009 Physical Review D 79124032 (Preprint 0812.2163)

[54] Abbott B P, Abbott R, Abbott T, Abernathy M, Acernese F, Ackley K, Adams C, Adams T, Addesso P, Adhikari R et al. 2019 Physical Review X 9011001 (Preprint 1805.11579)

[55] Douchin F and Haensel P 2001 Astronomy \& Astrophysics 380 151-167 (Preprint astro-ph/ 0111092)

[56] Banik S, Hempel M and Bandyopadhyay D 2014 The Astrophysical Journal Supplement 21422 (Preprint 1404.6173)

[57] Dietrich T, Khan S, Dudi R, Kapadia S J, Kumar P, Nagar A, Ohme F, Pannarale F, Samajdar A, Bernuzzi S, Carullo G, Del Pozzo W, Haney M, Markakis C, Pürrer M, Riemenschneider G, Setyawati Y E, Tsang K W and Van Den Broeck C 2019 Phys. Rev. D 99024029 (Preprint 1804.02235)

[58] Abbott B P, Abbott R, Abbott T D, Abraham S, Acernese F, Ackley K, Adams C, Adya V B, Affeldt et al. 2020 Classical and Quantum Gravity 37045006 (Preprint 1908.01012)

[59] Lattimer J and Schramm D 1976 Astrophys. J. 210549

[60] Lee W H and Ramirez-Ruiz E 2007 New J. Phys. 917 (Preprint astro-ph/0701874)

[61] Li L X and Paczynski B 1998 Astrophys. J. Lett. 507 L59 (Preprint astro-ph/9807272)

[62] Metzger B, Martinez-Pinedo G, Darbha S, Quataert E, Arcones A, Kasen D, Thomas R, Nugent P, Panov I and Zinner N 2010 Mon. Not. Roy. Astron. Soc. 4062650 (Preprint 1001. 5029)

[63] Nakar E and Piran T 2011 Nature 478 82-84 (Preprint 1102 .1020)

[64] Metzger B D and Berger E 2012 The Astrophysical Journal 74648

[65] Metzger B D and Zivancev C 2016 Mon. Not. Roy. Astron. Soc. 461 4435-4440 (Preprint 1605. 01060)

[66] Hallinan G et al. 2017 Science 358 1579-1583 (Preprint 1710.05435)

[67] Abbott B P et al. (LIGO Scientific, Virgo, Fermi GBM, INTEGRAL, IceCube, AstroSat Cadmium Zinc Telluride Imager Team, IPN, Insight-Hxmt, ANTARES, Swift, AGILE Team, 1M2H Team, Dark Energy Camera GW-EM, DES, DLT40, GRAWITA, Fermi-LAT, ATCA, ASKAP, Las Cumbres Observatory Group, OzGrav, DWF (Deeper Wider Faster Program), AST3, CAASTRO, VINROUGE, MASTER, J-GEM, GROWTH, JAGWAR, CaltechNRAO, TTU-NRAO, NuSTAR, Pan-STARRS, MAXI Team, TZAC Consortium, KU, Nordic Optical Telescope, ePESSTO, GROND, Texas Tech University, SALT Group, TOROS, BOOTES, MWA, CALET, IKI-GW Follow-up, H.E.S.S., LOFAR, LWA, HAWC, Pierre Auger, ALMA, Euro VLBI Team, Pi of Sky, Chandra Team at McGill University, DFN, ATLAS Telescopes, High Time Resolution Universe Survey, RIMAS, RATIR, SKA South Africa/MeerKAT) 2017 Astrophys. J. 848 L12 (Preprint 1710.05833)

[68] Rana J, Singhal A, Gadre B, Bhalerao V and Bose S 2017 The Astrophysical Journal 838108 (Preprint 1603.01689)

[69] Coughlin M W et al. 2018 MNRS 478 692-702 (Preprint 1803.02255)

[70] Ghosh S, Bloemen S, Nelemans G, Groot P J and Price L R 2016 Astronomy and Astrophysics 592 A82 (Preprint 1511.02673)

[71] Rana J, Anand S and Bose S 2019 The Astrophysical Journal 876104 (Preprint 1902. 08378)

[72] Abbott B P, Abbott R, Abbott T D, Abraham S, Acernese F, Ackley K, Adams C, Adhikari R X, Adya V B, Affeldt C and et al 2020 The Astrophysical Journal 892 L3 ISSN 2041-8213 URL http://dx.doi.org/10.3847/2041-8213/ab75f5

[73] Rana J and Mooley K P 2019 arXiv e-prints arXiv:1904.07335 (Preprint 1904.07335)

[74] Sathyaprakash B S and Dhurandhar S V 1991 Phys. Rev. D44(12) 3819-3834

[75] Blanchet L, Damour T, Iyer B R, Will C M and Wiseman A G 1995 Phys. Rev. Lett. 74 3515-3518 (Preprint gr-qc/9501027) 
[76] Blanchet L, Damour T, Esposito-Farese G and Iyer B R 2005 Phys. Rev. D71 124004 (Preprint gr-qc/0503044)

[77] Buonanno A, Iyer B R, Ochsner E, Pan Y and Sathyaprakash B S 2009 Phys. Rev. D80(8) 084043 (Preprint 0907.0700)

[78] Özel F and Freire P 2016 Ann. Rev. Astron. Astrophys. 54 401-440 (Preprint 1603.02698)

[79] Burgay M et al. 2003 Nature 426 531-533 (Preprint astro-ph/0312071)

[80] Zhu X, Thrane E, Oslowski S, Levin Y and Lasky P D 2018 Phys. Rev. D98 043002 (Preprint 1711.09226)

[81] Sachdev S et al. 2019 (Preprint 1901.08580)

[82] Hanna C et al. 2020 Phys. Rev. D101 022003 (Preprint 1901. 02227)

[83] Nitz A H, Dal Canton T, Davis D and Reyes S 2018 Phys. Rev. D98 024050 (Preprint 1805.11174)

[84] Adams T, Buskulic D, Germain V, Guidi G M, Marion F, Montani M, Mours B, Piergiovanni F and Wang G 2016 Class. Quant. Grav. 33175012 (Preprint 1512.02864)

[85] Chu Q 2017 Low-latency detection and localization of gravitational waves from compact binary coalescences $\mathrm{Ph} . \mathrm{D}$. thesis The University of Western Australia

[86] Singer L P and Price L R 2016 Phys. Rev. D 93(2) 024013 URL https://link.aps.org/doi/10. 1103/PhysRevD.93.024013

[87] Gorski K M et al. 2005 The Astrophysical Journal 622 759-771 (Preprint astro-ph/0409513)

[88] LIGO Scientific Collaboration V C 2017 GCN 21505 URL https://gcn.gsfc.nasa.gov/gcn3/ $21505 . \operatorname{gcn} 3$

[89] LIGO Scientific Collaboration V C 2017 GCN 21513 URL https://gcn.gsfc.nasa.gov/gcn3/ 21513.gcn3

[90] Cannon Ket al. 2012 Astrophys. J. 748136 (Preprint 1107.2665)

[91] Chu Q, Howell E J, Rowlinson A, Gao H, Zhang B, Tingay S J, Boër M and Wen L 2016 Monthly Notices of the Royal Astronomical Society 459 121-139 ISSN 0035-8711 (Preprint https: //academic.oup.com/mnras/article-pdf/459/1/121/8113983/stw576.pdf) URL https: //doi.org/10.1093/mnras/stw576

[92] Akcay S 2019 Annalen Phys. 5311800365 (Preprint 1808.10057)

[93] Nicholl M et al. 2017 Astrophys. J. 848 L18 (Preprint 1710.05456)

[94] Metzger B D 2017 Preprint: arXiv 1710.05931 (Preprint 1710.05931)

[95] Metzger B D and Piro A L 2014 Mon. Not. Roy. Astron. Soc. 439 3916-3930 (Preprint 1311.1519)

[96] Ciolfi R and Siegel D M 2015 Astrophys. J. 798 L36 (Preprint 1411.2015)

[97] Siegel D M and Ciolfi R 2016 Astrophys. J. 81915 (Preprint 1508.07939)

[98] Most E R and Philippov A A 2020 The Astrophysical Journal 893 L6 ISSN 2041-8213 URL http://dx.doi.org/10.3847/2041-8213/ab8196

[99] Totani T 2013 Pub. Astron. Soc. Jpn. 65 L12 (Preprint 1307.4985)

[100] Wang J S, Yang Y P, Wu X F, Dai Z G and Wang F Y 2016 Astrophys. J. 822 L7 (Preprint 1603.02014)

[101] Dokuchaev V I and Eroshenko Yu N 2017 (Preprint 1701.02492)

[102] Sachdev S, Magee R, Hanna C, Cannon K, Singer L, SK J R, Mukherjee D, Caudill S, Chan C, Creighton J D E and et al 2020 The Astrophysical Journal 905 L25 ISSN 2041-8213 URL http://dx.doi.org/10.3847/2041-8213/abc753

[103] Nitz A H, Schäfer M and Dal Canton T 2020 Astrophys. J. Lett. 902 L29 (Preprint 2009. 04439)

[104] Magee R, Chatterjee D, Singer L P, Sachdev S, Kovalam M, Mo G, Anderson S, Brady P, Brockill P, Cannon K, Canton T D, Chu Q, Clearwater P, Codoreanu A, Drago M, Godwin P, Ghosh S, Greco G, Hanna C, Kapadia S J, Katsavounidis E, Oloworaran V, Pace A E, Panther F, Patwary A, Pietri R D, Piotrzkowski B, Prestegard T, Rei L, Sreekumar A K, Szczepańczyk M J, Valsan V, Viets A and Wade M 2021 First demonstration of early warning gravitational wave alerts (Preprint 2102.04555)

[105] Cannon K, Caudill S, Chan C, Cousins B, Creighton J D E, Ewing B, Fong H, Godwin P, Hanna C, Hooper S, Huxford R, Magee R, Meacher D, Messick C, Morisaki S, Mukherjee D, Ohta H, Pace 
A, Privitera S, de Ruiter I, Sachdev S, Singer L, Singh D, Tapia R, Tsukada L, Tsuna D, Tsutsui T, Ueno K, Viets A, Wade L and Wade M 2020 Gstlal: A software framework for gravitational wave discovery (Preprint 2010.05082)

[106] BlackGEM 2020 The blackgem telescope array "https://astro.ru.nl/blackgem/" accessed 2020-07-17

[107] ZTF 2020 The zwicky transient facility "https ://www.ztf . caltech.edu/" accessed 2020-07-17

[108] Flaugher B, Diehl H T, Honscheid K, Abbott T M C, Alvarez O, Angstadt R, Annis J T, Antonik M, Ballester O, Beaufore L and et al 2015 The Astronomical Journal 150150 ISSN 1538-3881 URL http://dx.doi.org/10.1088/0004-6256/150/5/150

[109] LSST 2020 The large synoptic survey telescope "https://www. Isst.org/lsst/" accessed 2020$07-17$

[110] SWOPE 2020 The swope telescope "https://obs.carnegiescience.edu/swope" accessed 2020$07-17$

[111] Subaru 2020 The subaru telescope "https://subarutelescope.org/en/" accessed 2020-08-03

[112] Abbott B P et al. (LIGO Scientific Collaboration and Virgo Collaboration) 2016 Phys. Rev. Lett. 116 221101 (Preprint 1602.03841)

[113] Abbott B et al. (LIGO Scientific, Virgo) 2019 Phys. Rev. D 100104036 (Preprint 1903.04467)

[114] Abbott R et al. (LIGO Scientific, Virgo) 2020 (Preprint 2010.14529)

[115] Yunes N and Pretorius F 2009 Phys. Rev. D 80122003 (Preprint 0909.3328)

[116] Li T, Del Pozzo W, Vitale S, Van Den Broeck C, Agathos M, Veitch J, Grover K, Sidery T, Sturani R and Vecchio A 2012 Physical Review D 85082003

[117] Li T, Del Pozzo W, Vitale S, Van Den Broeck C, Agathos M, Veitch J, Grover K, Sidery T, Sturani R and Vecchio A 2012 Journal of Physics: Conference Series vol 363 (IOP Publishing) p 012028

[118] Agathos M, Del Pozzo W, Li T G F, Van Den Broeck C, Veitch J and Vitale S 2014 Phys. Rev. D 89 082001 (Preprint 1311.0420)

[119] Hughes S A and Menou K 2005 Astrophys. J. 623689 (Preprint astro-ph/0410148)

[120] Ghosh A, Del Pozzo W and Ajith P 2016 Phys. Rev. D 94104070 (Preprint 1505.05607)

[121] Ghosh A et al. 2016 Phys. Rev. D 94 021101(R) (Preprint 1602.02453)

[122] Mirshekari S, Yunes N and Will C M 2012 Phys. Rev. D 85024041 (Preprint 1110.2720)

[123] Yunes N, Yagi K and Pretorius F 2016 Phys. Rev. D 94084002 (Preprint 1603.08955)

[124] Will C M 1998 Phys. Rev. D 572061 (Preprint gr-qc/9709011)

[125] Calcagni G 2010 Phys. Rev. Lett. 104251301 (Preprint 0912.3142)

[126] Amelino-Camelia G 2002 Nature 418 34-35 (Preprint gr-qc/0207049)

[127] Horava P 2009 Phys. Rev. D 79084008 (Preprint 0901.3775 )

[128] Sefiedgar A, Nozari K and Sepangi H 2011 Phys. Lett. B 696 119-123 (Preprint 1012.1406)

[129] Kostelecký V and Mewes M 2016 Phys. Lett. B $757510-514$ (Preprint 1602.04782)

[130] Isi M, Pitkin M and Weinstein A J 2017 Phys. Rev. D 96042001 (Preprint 1703.07530)

[131] Abbott B P, Abbott R, Abbott T D, Acernese F, Ackley K, Adams C, Adams T, Addesso P, Adhikari $\mathrm{R} \mathrm{X}$ and et al (LIGO Scientific Collaboration and Virgo Collaboration) 2017 Phys. Rev. D 96(12) 122006 URL https://link .aps .org/doi/10.1103/PhysRevD .96.122006

[132] Callister T, Biscoveanu A, Christensen N, Isi M, Matas A, Minazzoli O, Regimbau T, Sakellariadou M, Tasson J and Thrane E 2017 Phys. Rev. X 7041058 (Preprint 1704.08373)

[133] Abbott B P et al. (LIGO Scientific, Virgo) 2018 Phys. Rev. Lett. 120201102 (Preprint 1802. 10194)

[134] Nishizawa A, Taruya A, Hayama K, Kawamura S and Sakagami M a 2009 Phys. Rev. D 79082002 (Preprint 0903.0528)

[135] Isi M and Weinstein A J 2017 (Preprint 1710.03794)

[136] Pang P T, Lo R K, Wong I C, Li T G and Van Den Broeck C 2020 Phys. Rev. D 101104055 (Preprint 2003.07375)

[137] Abbott B, Abbott R, Abbott T, Acernese F, Ackley K, Adams C, Adams T, Addesso P, Adhikari R, Adya V and et al 2019 Physical Review Letters 123 ISSN 1079-7114 URL http: //dx.doi .org/ 10.1103/PhysRevLett. 123.011102 
[138] Abbott B P et al. (LIGO Scientific Collaboration and Virgo Collaboration) 2017 Phys. Rev. Lett. 119(14) 141101 URL https://link.aps .org/doi/10.1103/PhysRevLett.119.141101

[139] Chatziioannou K, Yunes N and Cornish N 2012 Physical Review D 86 ISSN 1550-2368 URL http://dx.doi.org/10.1103/PhysRevD.86.022004

[140] Takeda H, Morisaki S and Nishizawa A 2021 Physical Review D 103 ISSN 2470-0029 URL http://dx.doi.org/10.1103/PhysRevD.103.064037

[141] Hagihara Y, Era N, Iikawa D, Nishizawa A and Asada H 2019 Physical Review D 100 ISSN 24700029 URL http://dx.doi.org/10.1103/PhysRevD.100.064010

[142] Abbott B et al. (LIGO Scientific, Virgo) 2019 Phys. Rev. D 100104036 (Preprint 1903.04467)

[143] Veitch J et al. 2015 Phys. Rev. D91 042003 (Preprint 1409.7215)

[144] Hayama K and Nishizawa A 2013 Phys. Rev. D 87(6) 062003 URL https://link.aps.org/doi/ 10.1103/PhysRevD.87.062003

[145] Regimbau T and Mandic V 2008 Classical and Quantum Gravity 25184018 URL https://doi. org/10.1088\%2F0264-9381\%2F25\%2F18\%2F 184018

[146] Regimbau T 2011 Research in Astronomy and Astrophysics 11 369-390 URL https://doi.org/ 10. $1088 \% 2 \mathrm{~F} 1674-4527 \% 2 \mathrm{~F} 11 \% 2 \mathrm{~F} 4 \% 2 \mathrm{~F} 001$

[147] Abbott R et al. (LIGO Scientific, Virgo, KAGRA) 2021 (Preprint 2101.12130)

[148] Callister T, Fishbach M, Holz D and Farr W 2020 Astrophys. J. Lett. 896 L32 (Preprint 2003.12152)

[149] Mitra S, Dhurandhar S, Souradeep T, Lazzarini A, Mandic V, Bose S and Ballmer S 2008 Phys. Rev. D 77(4) 042002 URL https://link.aps.org/doi/10.1103/PhysRevD . 77.042002

[150] Talukder D, Mitra S and Bose S 2011 Phys. Rev. D 83(6) 063002 URL https://link.aps.org/ doi/10.1103/PhysRevD.83.063002

[151] Ain A, Suresh J and Mitra S 2018 Phys. Rev. D 98024001 (Preprint 1803.08285)

[152] Suresh J, Ain A and Mitra S 2020 (Preprint 2011.05969)

[153] van der Walt S, Colbert S C and Varoquaux G 2011 Comput. Sci. Eng. 13 22-30 (Preprint 1102.1523)

[154] Virtanen P et al. 2020 Nature Meth. (Preprint 1907.10121)

[155] Hunter J D 2007 Computing in Science \& Engineering 9 90-95

[156] Robitaille T P et al. (Astropy) 2013 Astron. Astrophys. 558 A33 (Preprint 1307.6212)

[157] Price-Whelan A et al. 2018 Astron. J. 156123 (Preprint 1801. 02634)

[158] Kluyver T, Ragan-Kelley B, Pérez F, Granger B, Bussonnier M, Frederic J, Kelley K, Hamrick J, Grout J, Corlay S, Ivanov P, Avila D, Abdalla S, Willing C and development team J 2016 Positioning and Power in Academic Publishing: Players, Agents and Agendas ed Loizides F and Scmidt B (Netherlands: IOS Press) pp 87-90 URL https: //eprints . soton. ac.uk/403913/

[159] Higson E, Handley W, Hobson M and Lasenby A 2019 Statistics and Computing 29 891-913 (Preprint 1704.03459)

[160] Hoy C and Raymond V 2020 (Preprint 2006.06639) 